\title{
Magnetic Attitude Control for Satellites in Polar or Sun-Synchronous Orbits
}

\author{
Javier Cubas, ${ }^{*}$ Assal Farrahi, ${ }^{\dagger}$ and Santiago Pindado ${ }^{\ddagger}$ \\ Universidad Politécnica de Madrid, 28040 Madrid, Spain
}

\begin{abstract}
In this work, a new law for magnetic control of satellites in near-polar orbits is presented. This law has been developed for the UMPSat-2 microsatellite, which has been designed and manufactured by Universidad Politécnica de Madrid, Madrid. The control law is a modification of the $B$-dot strategy that enables the satellite to control the rotation rate. Besides, the satellite's equilibrium state is characterized by having the rotation axis perpendicular to the orbit's plane. The control law described in the present work only needs magnetometers and magnetorquers, as sensors and actuators, respectively, to carry out a successful attitude control on the spacecraft. A description of the analysis is included. Performance and applicability of the proposed method have been demonstrated by control dynamics together with Monte Carlo techniques and by implementing the control law in the UPMSat-2 mission simulator. Results show good performance in terms of acquisition and stability of the satellite rotation rate and orientation with respect to its orbit's plane.
\end{abstract}

\section{Nomenclature}

$E_{\text {kin }} \quad=$ kinetic energy, $\mathrm{J}$

$\hat{\boldsymbol{e}}_{x}, \hat{\boldsymbol{e}}_{y}, \hat{\boldsymbol{e}}_{z}=$ unitary vectors of a frame

$F_{E} \quad=$ Earth-centered, Earth-fixed frame

$F_{i} \quad=$ Earth-centered inertial frame

$F_{s} \quad=$ spacecraft fixed frame

$F_{0} \quad=$ auxiliary reference frame parallel to orbit plane

$F_{1} \quad=$ first auxiliary frame

$F_{2} \quad=$ second auxiliary frame

$\boldsymbol{h}=$ angular momentum vector, $\mathrm{kg} \cdot \mathrm{m}^{2} \cdot \mathrm{s}^{-1} \cdot \mathrm{rad}$

$\boldsymbol{I}=$ spacecraft matrix of inertia along its principal axes, $\mathrm{kg} \cdot \mathrm{m}^{2}$

$I_{\perp} \quad=$ inertia moment of spacecraft around $x_{s}$ and $y_{s}$ axes, $\mathrm{kg} \cdot \mathrm{m}^{2}$

$I_{\|} \quad=\quad$ inertia moment of spacecraft around $z_{s}$ axis, $\mathrm{kg} \cdot \mathrm{m}^{2}$

$i \quad=$ inclination of orbit, rad

$k \quad=\quad$ control gain, $\mathrm{A} \cdot \mathrm{m}^{2} \cdot \mathrm{T}^{-1} \cdot \mathrm{s}$

$\boldsymbol{m} \quad=$ magnetic dipole moment vector of control, $\mathrm{A} \cdot \mathrm{m}^{2}$

$\boldsymbol{m}_{E} \quad=\quad$ magnetic dipole moment vector of Earth, $\mathrm{A} \cdot \mathrm{m}^{2}$

$R=$ distance to Earth center, $\mathrm{m}$

$\boldsymbol{R}=$ vector position with respect to Earth center, $\mathrm{m}$

$R_{E} \quad=\quad$ Earth average radius, $\mathrm{m}$

$R_{0} \quad=$ orbit radius, $\mathrm{m}$

$\boldsymbol{S}(\cdot) \quad=\quad$ skew symmetric matrix

$\boldsymbol{T}=$ external torque acting on the spacecraft, $\mathrm{N} \cdot \mathrm{m}$

$T_{\text {coil }}=$ magnetic control torque, $\mathrm{N} \cdot \mathrm{m}$

Received 14 May 2014; revision received 3 May 2015; accepted for publication 4 May 2015; published online 31 July 2015 . Copyright $@ 2015$ by the American Institute of Aeronautics and Astronautics, Inc. All rights reserved. Copies of this paper may be made for personal or internal use, on condition that the copier pay the $\$ 10.00$ per-copy fee to the Copyright Clearance Center, Inc., 222 Rosewood Drive, Danvers, MA 01923; include the code $1533-3884 / 15$ and $\$ 10.00$ in correspondence with the CCC.

*Instituto Universitario de Microgravedad "Ignacio Da Riva"/Universidad Politécnica de Madrid (IDR/UPM), ETSIAE, Plaza Cardenal Cisneros Número 3; j.cubas@upm.es.

†Instituto Universitario de Microgravedad "Ignacio Da Riva"/Universidad Politécnica de Madrid (IDR/UPM), ETSIAE, Plaza Cardenal Cisneros Número 3; assal.farrahi@upm.es.

"Instituto Universitario de Microgravedad "Ignacio Da Riva"/Universidad Politécnica de Madrid (IDR/UPM) \& Departamento de Sistemas Aeroespaciales, Transporte Aéreo y Aeropuertos, ETSIAE, Plaza Cardenal Cisneros Número 3; santiago.pindado@upm.es.

\begin{tabular}{|c|c|c|}
\hline $\boldsymbol{T}_{\text {dist }}$ & $=$ & disturbance torque, $\mathrm{N} \cdot \mathrm{m}$ \\
\hline$t$ & $=$ & time, $\mathrm{s}$ \\
\hline$u$ & $=$ & argument of latitude in circular orbit, rad \\
\hline$\alpha$ & $=$ & angle between ${ }^{0} \dot{\boldsymbol{B}}(t) \times{ }^{0} \boldsymbol{B}(t)$ and $Z_{0}$, rad \\
\hline$\gamma$ & $=$ & angle between $z_{s}$ and $Z_{0}$, rad \\
\hline$\Theta_{T}$ & $=$ & rotation angle between $F_{E}$ frame and $F_{i}$, rad \\
\hline$\theta$ & $=$ & second Euler angle, rad \\
\hline$\theta_{m}^{\prime}$ & $=$ & coelevation angle of dipole direction in $F_{E}$, rad \\
\hline$\tau$ & $=$ & attitude correction torque, $\mathrm{N} \cdot \mathrm{m}$ \\
\hline$\phi$ & $=$ & first Euler angle, rad \\
\hline$\phi_{m}^{\prime}$ & $=$ & east longitude angle of dipole direction in $F_{E}$, rad \\
\hline$\psi$ & $=$ & third Euler angle, rad \\
\hline$\Omega_{0}$ & $=$ & orbital rotation rate, $\mathrm{rad} / \mathrm{s}$ \\
\hline$\Omega_{02}$ & $=$ & angular velocity of $F_{2}$ with respect to $F_{0}, \mathrm{rad} / \mathrm{s}$ \\
\hline$\omega$ & $=$ & satellite angular velocity vector, $\mathrm{rad} / \mathrm{s}$ \\
\hline$\omega_{d}$ & $=$ & $\begin{array}{l}\text { desired angular velocity, parameter of the control, } \\
\mathrm{rad} / \mathrm{s}\end{array}$ \\
\hline
\end{tabular}

Superscripts

$\begin{array}{lll}E & =\text { vector components expressed in } F_{E} \text { frame } \\ i & =\text { vector components expressed in } F_{i} \text { frame } \\ s & =\text { vector components expressed in } F_{s} \text { frame } \\ 0 & =\text { vector components expressed in } F_{0} \text { frame } \\ 1 & =\text { vector components expressed in } F_{1} \text { frame } \\ 2 & =\text { vector components expressed in } F_{2} \text { frame }\end{array}$

\section{Introduction}

Q INCE the successful operation of the magnetic controllers of the $\checkmark$ early space programs [1], the magnetic attitude control strategy has turned into an attractive alternative for the attitude control of those small satellites with not-too-demanding orientation requirements [2-4]. This strategy significantly saves overall power, weight, cost, and complexity of the system compared to other attitude control strategies, thanks to not having any propellant or moving parts. For the same reason of simplicity, the risk of failure of this strategy is reduced, and the system is more reliable. This fact makes magnetic attitude control very suitable for micro-, nano-, and picosatellites operating in low Earth orbits (LEOs).

As is well known, the magnetic control can be either active or passive. In the passive magnetic attitude control, elements that do not require any power supply, like hysteresis rods or a permanent magnet, are used [4-6]. In active magnetic attitude control, magnetorquers are used as actuators. These elements can be regulated by varying their input currents, and this allows the implementation of closed-loop feedback laws, based on the sensors' measurements $[7,8]$. 
However, despite its advantages, a spacecraft controlled with magnetic actuators would always be an underactuated system [5], as the magnetic torque, regardless of the number of the magnetic rods, can only be generated perpendicular to the local Earth's magnetic field. Nevertheless, if satellite orbit inclination with respect to the geomagnetic equator is enough, the magnetic field vector periodically changes its direction with respect to the inertial reference frame, and as a result, it is possible (at least on average over the orbital period) to apply torques in any direction [9-11]. Therefore, if closedloop dynamics is sufficiently slow, linear conventional proportional derivative control law can be applied [12]. On the other hand, it should be also pointed out that in case of large rotations or high angular rates (e.g., for initial satellite detumbling or spinning spacecraft) high nonlinearities are involved, and as a consequence, nonlinear controls are necessary instead of the linear ones.

Leaving aside some recent advances in nonlinear predictive control [13], it should be underlined that one of the most used nonlinear strategies is the well-known $B$-dot control law. Apart from its fundamental role for spacecraft initial attitude acquisition, one of the major advantages of $B$-dot is its simplicity, allowing fully magnetic device-based control with solely magnetic instruments. This makes this strategy appropriate for small satellites [14-16]. The challenge of fully magnetic device-based control is the lack of attitude observation by magnetometers, which does not allow typical linear control strategies. Thus, for a long time, $B$-dot control law was the only strategy available. In this control law, the supplied magnetic dipole moment vector is proportional to the derivative of the magnetic field, and this results in a dissipative effect that decreases the angular velocity of the spacecraft. This dissipation remains for as long as the rate of variation of the magnetic field along the orbit is negligible, when compared to the rate of variation caused by the spacecraft rotation [12]. This implies that, using this control law, the angular velocity of the satellite cannot be stabilized at a particular chosen value, but depending on the inclination of its orbit, the angular velocity is reduced up to a limit. Obviously, this lack of control over the satellite rotational rate could be a problem in terms of thermal control, as some part of the satellite surface might be overexposed to the sun radiation if the stabilized rotation rate is too low. Another disadvantage of the $B$-dot strategy is the lack of control regarding the final attitude of the satellite (once stabilized), which depends on its initial condition. Consequently, the magnetic attitude control systems that are exclusively based on this strategy normally include a permanent magnet, making one of the satellite axes remain permanently aligned with the geomagnetic field [17].

Because of the aforementioned drawbacks, in recent years, some studies have been carried out to find some more efficient alternatives for $B$-dot control law. Some of these $B$-dot-like developments might overcome the weaknesses of the $B$-dot control law, e.g., allowing a reduction in the angular rates of the satellite to zero [18], or the acquisition of a desired spin condition along a prescribed axis in inertial frame [19], but requiring direct measurement (or estimation) of angular velocity components. This is, as already mentioned, not possible to evaluate directly from magnetometers' data, making fully magnetic device-based control not possible. Nevertheless, it is possible to compare magnetometers measurements with geomagnetic models on board in order to make some attitude estimations, which allows the design and development of fully magnetic device-based control [20]. However, that increases the complexity of the control and the data stored on the onboard satellite computer and requires certain additional information (or estimations), such as the exact position of the satellite.

In the present work, a new nonlinear magnetic attitude control that, to some extent, overcomes the aforementioned deficiencies of $B$-dot control is described. The control subsystem presented has a very simple design, it does not include any model or information stored onboard, and it only uses magnetorquers as actuators and magnetometers as sensors. The programmed control law is based on a modification of the $B$-dot strategy. The main advantage of this modification is that it enables effective control of the satellite angular rotation rate, which is not possible using the traditional $B$-dot control law. Therefore, it can represent an improvement from the thermal control point of view. Additionally, the proposed control law will force a terminal attitude such that the rotation axis aligns with the orbit normal. This effect has already been described for spacecraft of the momentum-bias variety with $B$-dot control law [21]. The proposed control law obtains the same result without using any reaction wheel.

In Sec. II, a description of the UPMSat-2 satellite mission and the general configuration of its attitude, control, and determination subsystem is included, whereas in Sec. III, the general dynamics of a satellite with magnetic control is introduced. In Sec. IV, the proposed control law is presented, and the rotation rate correction is explained. In Sec. V, the attitude evolution and stabilized solution are described. In Sec. VI, the control law is analyzed by means of the Monte Carlo technique and by including it in the attitude simulator developed for the UPMSat-2 mission. Results from the numerical simulation are included in this section. The paper's conclusions are summarized in Sec. VII.

\section{UPMSat-2 Satellite}

The control presented in this paper was designed for the UPMSat-2 satellite, which is scheduled to be launched during 2015 . The mission characteristics have impacted the attitude control approach and design. These characteristics are summarized in the following.

UPMSat-2 is a $50 \mathrm{~kg}$ microsatellite completely developed at Instituto Universitario de Microgravedad "Ignacio Da Riva" (IDR/ UPM) of Universidad Politécnica de Madrid (Madrid, Spain), for a 2 year LEO mission, orbiting in a sun-synchronous noon/midnight orbit, at $700 \mathrm{~km}$ altitude [22] (see Table 1). This satellite mission is preceded by UPMSat-1 launched in 1995 by the same research group [23].

The Attitude, Control, and Determination Subsystem (ACDS) of UPMSat-2 includes three ZARM Technik AG magnetorquers, and two SSBV Aerospace \& Technology Group Space and Ground Systems magnetometers. Obviously, one of the magnetometers would be sufficient, with a second one being included to increase the subsystem reliability. Furthermore, the information from a third magnetometer, by Bartington Instruments, present onboard as payload, will be available for the ADCS during the mission. As the UPMSat-2 ACDS only includes magnetic sensors and actuators, the control law developed is purely magnetic.

A picture of the UPMSat-2 is included in Fig. 1. The picture shows the solar panels (built with eight SPVS-5S modules by SELEX Galileo, attached at lateral faces $+x_{s},-x_{s},+y_{s}$, and $-y_{s}$, and half of one, formed by four SPVS-5S modules, at the top one, $+z_{s}$ ) can be clearly observed. Also, a sketch indicating the attitude of the satellite in the selected almost-polar sun-synchronous orbit has been included. Taking into account the aforementioned orbit, it can be said that the solar radiation will be almost perpendicular to the aforementioned rotation axis, allowing an easier thermal control thanks to the rotation. Besides, as the solar radiation falls directly to the side panels of the satellite, quite a high power supply is obtained through the solar panels (three different satellite attitudes within the selected orbit were analyzed for the UPMSat-2, the one described in the present work having the best result in terms of power supply [24]). The solar cells allocated in face $+z_{s}$ are installed to produce some power supply in case of attitude perturbations or uncontrolled transient situations.

\section{Dynamics of a Satellite with Magnetic Control}

\section{A. Reference Frames}

In the present work, the following reference frames (i.e., coordinates systems), are considered:

Table 1 General characteristics of the UPMSat-2 satellite

\begin{tabular}{lc}
\hline \hline Characteristic & Description \\
\hline Dimensions, $\mathrm{m}$ & $0.50 \times 0.50 \times 0.60$ \\
Mass, $\mathrm{kg}$ & 550 \\
Moment of inertia, $z_{s}$ axis, $\mathrm{kg} \cdot \mathrm{m}^{2}$ & 1.833 \\
Moment of inertia, $x_{s}$ axis, $\mathrm{kg} \cdot \mathrm{m}^{2}$ & 2.543 \\
Moment of inertia, $y_{s}$ axis, $\mathrm{kg} \cdot \mathrm{m}^{2}$ & 2.525 \\
Orbital altitude, $\mathrm{km}$ & 700 \\
Period, min & $\approx 98$ (eclipse: 36) \\
\hline \hline
\end{tabular}



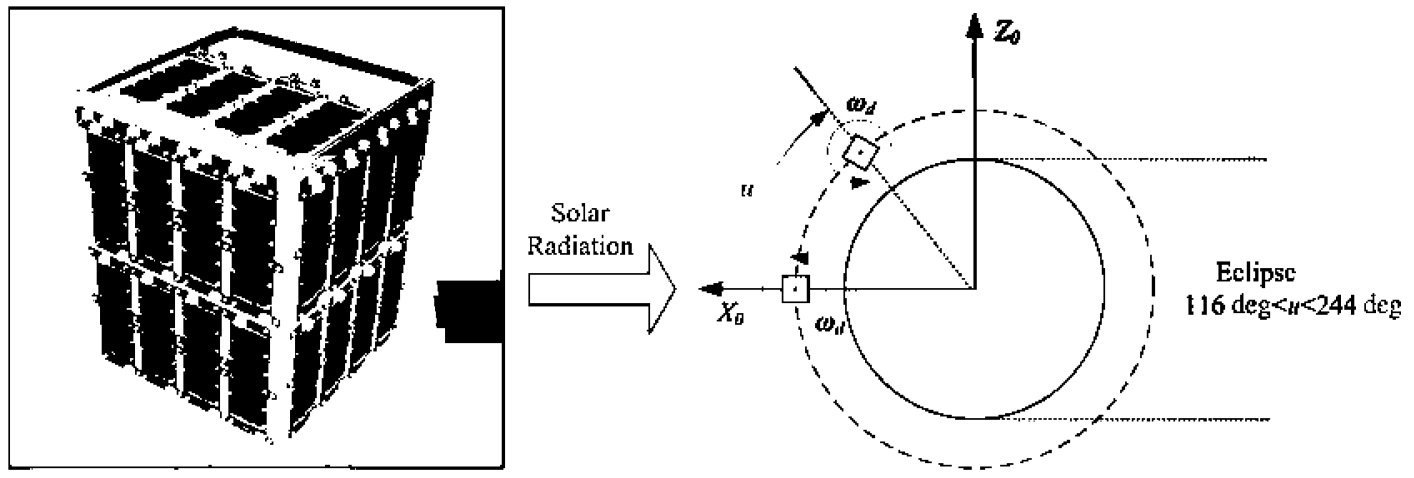

Fig. 1 Left: UPMSat-2 picture. Right: Sketch of the satellite in the selected orbit.

1) $F_{i}$ Earth-centered inertial reference frame; with its origin coinciding with the center of the Earth, $X_{i}$ pointing toward Vernal Equinox (Aries point) in the positive direction, the $Z_{i}$ axis parallel to the Earth's rotational axis pointing toward north, and the $Y_{i}$ axis completing the right-hand Cartesian frame.

2) $F_{E}$ Earth-centered, Earth-fixed reference frame; with its origin coinciding with the center of the Earth, the $z_{E}$ axis parallel to the rotational axis of the Earth in the positive direction, the $x_{E}$ axis pointing to the geographical zero latitude and longitude point (Equator crossing the Greenwich meridian), and the $y_{E}$ axis completing the right-hand Cartesian frame.

3) $F_{0}$ (auxiliary reference frame parallel to orbit plane); with its origin coinciding with the center of the Earth, the $X_{0}$ axis pointing toward the ascending node of the orbit, $Z_{0}$ perpendicular to the orbit toward the positive direction of the rotational axis of the satellite in orbit, and the $Y_{0}$ axis completing the right-hand Cartesian frame.

4) $F_{s}$ (body reference frame); with its origin coinciding with the center of mass of the satellite, the axes coinciding with the principle axes of the body, and $z_{s}$ parallel to the axis with the maximum inertia.

For some orbits, the $F_{0}$ frame can be considered inertial for short periods of time. In the particular case of sun-synchronous orbits, where the precession phenomenon is slow (about $1 \mathrm{deg}$ per day), this particular movement can be left aside for analysis within short periods of around $24 \mathrm{~h}$ or less. Therefore, in this work, $F_{0}$ is considered as an inertial coordinates system and used as the reference coordinates system. Besides, the $X_{0}$ axis is considered to match the $X_{i}$ axis. Although this reference frame is not commonly chosen by other authors, in the present work, it has been chosen to clearly show the desired effect of getting the rotation axis perpendicular to the orbit's plane.

\section{B. Attitude Dynamics}

Using the Euler equation, the dynamic model of a rigid satellite, expressed in the body reference frame $F_{s}$ is [25]

$$
I^{s} \dot{\omega}=-{ }^{s} \omega \times I^{s} \omega+{ }^{s} T_{\text {coil }}+{ }^{s} T_{\text {dist }}
$$

where $\boldsymbol{I}$ is the inertia matrix of the satellite in the aforementioned body reference frame, ${ }^{s} \omega$ is the angular velocity vector of the satellite with respect to the inertial reference frame, ${ }^{s} \boldsymbol{T}_{\text {coil }}$ is the control magnetic torque, and ${ }^{s} \boldsymbol{T}_{\text {dist }}$ is the torque due to environmental disturbances.

In the present work, a satellite almost axisymmetric around its $z_{s}$ axis, such as the UPMSat-2, is considered. The inertia matrix $I$ of such a satellite is defined (in the body frame $F_{s}$ ) as

$$
\boldsymbol{I}=\left[\begin{array}{ccc}
I_{\perp} & 0 & 0 \\
0 & I_{\perp} & 0 \\
0 & 0 & I_{\|}
\end{array}\right]
$$

The control torque $\boldsymbol{T}_{\text {coil }}$ is the result of the interaction between the magnetic dipole moment of the magnetorquers (actuators) $\boldsymbol{m}$ and the geomagnetic field $\boldsymbol{B}$. It can be expressed through the equation

$$
\boldsymbol{T}_{\text {coil }}(t)=\boldsymbol{m}(t) \times B(t)=\boldsymbol{S}[\boldsymbol{B}(t)] \boldsymbol{m}(t)
$$

where, as it can be appreciated, the resulting control torque is perpendicular to the magnetic field and $\boldsymbol{S}(\cdot)$ is the operator in matrix form equivalent to the vector product operation:

$$
\boldsymbol{S}(\boldsymbol{B})=-\boldsymbol{B} \times=\left[\begin{array}{ccc}
0 & B_{z} & -B_{y} \\
-B_{z} & 0 & B_{x} \\
B_{y} & -B_{x} & 0
\end{array}\right]
$$

Through the mathematical analysis presented in this work, the torque caused by environmental disturbances ${ }^{s} \boldsymbol{T}_{\text {dist }}$ included in expression (1) is not taken into account. Nevertheless, the most important perturbations for a satellite in a low Earth orbit, including aerodynamic and solar perturbations, have been included in the simulations carried out for validation of the proposed analytical formulation (in Sec. VI).

\section{Magnetic Field of Earth}

The geomagnetic field $\boldsymbol{B}$ can be approximated with the magnetic dipole model [19], as the magnetic field is similar to the one generated by a magnetic dipole correctly positioned and oriented at the center of the Earth,

$$
\boldsymbol{B}(\boldsymbol{R})=\frac{1}{R^{3}}\left[3\left(\boldsymbol{m}_{E} \cdot \frac{\boldsymbol{R}}{R}\right) \frac{\boldsymbol{R}}{R}-\boldsymbol{m}_{E}\right]
$$

where $\boldsymbol{R}$ is the vector position from the center of the Earth, $R=\|\boldsymbol{R}\|$, and $\boldsymbol{m}_{E}$ is the Earth approximated magnetic dipole in those axes. The dipole $\boldsymbol{m}_{E}$ can be expressed in $F_{E}$ reference as

$$
{ }^{E} \boldsymbol{m}_{E}=R_{E}{ }^{3} H_{0}\left[\begin{array}{c}
\sin \theta_{m}^{\prime} \cos \phi_{m}^{\prime} \\
\sin \theta_{m}^{\prime} \sin \phi_{m}^{\prime} \\
\cos \theta_{m}^{\prime}
\end{array}\right]
$$

where $R_{E}{ }^{3} H_{0}$ is the dipole strength [26],

$$
R_{E}^{3} H_{0}=7.77 \times 10^{15} \mathrm{~Wb} \cdot \mathrm{m}
$$

and $\theta_{m}^{\prime}$ and $\phi_{m}^{\prime}$ are, respectively, the latitude and longitude of the dipole direction in $F_{E}$ [26]:

$$
\begin{aligned}
& \theta_{m}^{\prime}=2.9673 \mathrm{rad} \\
& \phi_{m}^{\prime}=1.8812 \mathrm{rad}
\end{aligned}
$$

Taking the Earth's rotation into account, the magnetic dipole can be expressed in $F_{i}$ as

$$
{ }^{i} \boldsymbol{m}_{E}=R_{E}{ }^{3} H_{0}\left[\begin{array}{c}
\cos \Theta_{T} \sin \theta_{m}^{\prime} \cos \phi_{m}^{\prime}-\sin \Theta_{T} \sin \theta_{m}^{\prime} \sin \phi_{m}^{\prime} \\
\sin \Theta_{T} \sin \theta_{m}^{\prime} \cos \phi_{m}^{\prime}+\cos \Theta_{T} \sin \theta_{m}^{\prime} \sin \phi_{m}^{\prime} \\
\cos \theta_{m}
\end{array}\right]
$$

where $\Theta_{T}$ is the rotation angle between the $F_{E}$ frame and $F_{i}$ frame. 
The geomagnetic field in the $F_{0}$ reference frame can then be calculated by using Eq. (5) for the position of the satellite in the orbit ${ }^{0} \boldsymbol{R}$,

$$
{ }^{0} \boldsymbol{R}=R_{0}\left[\begin{array}{c}
\cos \left(u_{0}+\Omega_{o} t\right) \\
\sin \left(u_{0}+\Omega_{o} t\right) \\
0
\end{array}\right]
$$

and magnetic dipole vector $\boldsymbol{m}_{E}$, expressed in the $F_{0}$ reference frame:

$$
\begin{gathered}
{ }^{0} \boldsymbol{m}_{E}=R_{E}{ }^{3} H_{0}\left[\begin{array}{ccc}
1 & 0 & 0 \\
0 & \cos i & -\sin i \\
0 & \sin i & \cos i
\end{array}\right] \\
{\left[\begin{array}{c}
\cos \Theta_{T} \sin \theta_{m}^{\prime} \cos \phi_{m}^{\prime}-\sin \Theta_{T} \sin \theta_{m}^{\prime} \sin \phi_{m}^{\prime} \\
\sin \Theta_{T} \sin \theta_{m}^{\prime} \cos \phi_{m}^{\prime}+\cos \Theta_{T} \sin \theta_{m}^{\prime} \sin \phi_{m}^{\prime} \\
\cos \theta_{m}
\end{array}\right]}
\end{gathered}
$$

\section{Rotation Rate Correction}

The proposed law of control for the magnetic moment in body frame $F_{s}$ is defined as follows:

Corrigendum to the published paper:

$$
{ }^{s} \boldsymbol{m}=-k\left({ }^{s} \dot{\boldsymbol{B}}+{ }^{s} \boldsymbol{\omega}_{d} \times{ }^{s} \boldsymbol{B}\right)
$$

In the previous equation, ${ }^{s} m$ is the magnetic moment generated by magnetorquers, ${ }^{s} \boldsymbol{B}$ is the Earth magnetic field, ${ }^{s} \dot{\boldsymbol{B}}$ is the derivative of the Earth's magnetic field with respect to the same reference frame, $k$ is the control gain, and ${ }^{s} \omega_{d}$ is the desired angular rate, which is $\omega_{d} \gg \Omega_{o}$ and parallel to the axisymmetric principal axis $\left[z_{s}\right.$ as established by expression (2)]. The derivative of the magnetic field in this reference can be rewritten as [27]

$$
{ }^{s} \dot{B}={ }_{0}^{s} A^{0} \dot{B}+{ }^{s} B \times{ }^{s} \omega
$$

where ${ }_{0}^{s} \boldsymbol{A}$ is the transformation matrix from the inertial frame $F_{0}$ to the body frame $F_{s}$.

Considering the magnetic dipole indicated in Eq. (12), the control torque can be expressed as

$$
{ }^{s} \boldsymbol{T}_{\text {coil }}(t)=-k\left({ }^{s} \dot{\boldsymbol{B}}+{ }^{s} \boldsymbol{\omega}_{d} \times{ }^{s} \boldsymbol{B}\right) \times{ }^{s} \boldsymbol{B}
$$

Besides, bearing in mind the control torque explained in Eq. (14) and leaving the environmental disturbance torque aside, the dynamic equation of the satellite (1) results in

$$
\boldsymbol{I}^{s} \dot{\boldsymbol{\omega}}=-{ }^{s} \boldsymbol{\omega} \times \boldsymbol{I}^{s} \boldsymbol{\omega}-k\left({ }^{s} \dot{\boldsymbol{B}}+{ }^{s} \boldsymbol{\omega}_{d} \times{ }^{s} \boldsymbol{B}\right) \times{ }^{s} \boldsymbol{B}
$$

By taking into account expression (13) and reorganizing the different terms, Eq. (15) turns into the following equation:

$$
\boldsymbol{I}^{s} \dot{\boldsymbol{\omega}}=-k^{s} \boldsymbol{B} \times\left({ }^{s} \boldsymbol{\omega}-{ }^{s} \boldsymbol{\omega}_{d}\right) \times{ }^{s} \boldsymbol{B}-k_{0}^{s} \boldsymbol{A}\left({ }^{0} \dot{\boldsymbol{B}} \times{ }^{0} \boldsymbol{B}\right)-{ }^{s} \boldsymbol{\omega} \times \boldsymbol{I}^{s} \boldsymbol{\omega}
$$

Given an arbitrary value of the satellite angular rotation rate ${ }^{s} \omega$, different from the target one ${ }^{s} \omega_{d}$, it is reasonable to assume the following condition:

$$
\left\|{ }^{s} \omega-{ }^{s} \omega_{d}\right\| \gg \Omega_{o}
$$

Otherwise, it could be said that rotation rate correction phase would have finished, given that the target rotation rate is much greater than the orbital rotation rate, i.e., $\omega_{d} \gg \Omega_{o}$.

Comparing the order of magnitude of the different terms in expression (17) [which can be estimated with Eqs. (5) and (6)], it can be appreciated that the second term of the right side of the aforementioned expression is negligible when compared to the former one. This difference in the order of magnitude of the terms is caused as the variation of the magnetic field (in satellite axes) due to its rotational rate about its center of mass is much greater than the variation produced by its movement along the orbit since ${ }^{0} \boldsymbol{B}$ in the inertial axes varies at angular rate $2 \Omega_{o}$. Therefore, at higher angular rates of the satellite about its center of mass, the aforementioned Eq. (16) can be reduced to

$$
\boldsymbol{I}^{s} \dot{\boldsymbol{\omega}}=-k^{s} \boldsymbol{B} \times\left({ }^{s} \boldsymbol{\omega}-{ }^{s} \boldsymbol{\omega}_{d}\right) \times{ }^{s} \boldsymbol{B}-{ }^{s} \boldsymbol{\omega} \times \boldsymbol{I}^{s} \boldsymbol{\omega}
$$

This equation is similar to the one obtained for the well-known Bdot attitude control [27], and as a result, similar techniques can be used to prove that it is asymptotically stable up to the ${ }^{s} \omega={ }^{s} \omega_{d}$ angular velocity. Using the criteria of Lyapunov [28], we have the following:

Let $x=0$ be an equilibrium point for $\dot{x}=f(x)$ and $D \subset R^{n}$ be a domain containing $x=0$. Let the Lyapunov candidate function $V: D \rightarrow R$ be a continuously differentiable function such that

$$
V(0)=0 \quad V(x)>0 \quad \text { in } D-\{0\}
$$

Then, $x=0$ is stable if

$$
\dot{V}(x) \leq 0 \text { in } D
$$

and asymptotically stable if

$$
\dot{V}(x)<0 \quad \text { in } D-\{0\}
$$

If ${ }^{s} \omega^{*}={ }^{s} \omega-{ }^{s} \omega_{d}$ is defined as an auxiliary variable and the kinetic energy equation based on that rotational speed is considered as a candidate Lyapunov function,

$$
E_{\mathrm{kin}}\left({ }^{s} \boldsymbol{\omega}^{*}\right)=\frac{1}{2}{ }^{s} \boldsymbol{\omega}^{* T} \boldsymbol{I}^{s} \boldsymbol{\omega}^{*}
$$

an equation that satisfies the aforementioned requirements (24) is obtained:

$$
E_{\mathrm{kin}}(0)=0 \quad E_{\mathrm{kin}}\left({ }^{s} \omega^{*}\right)>0 \quad \forall{ }^{s} \omega^{*} \neq 0
$$

The derivative of the candidate function can be then expressed as

$$
\dot{E}_{\mathrm{kin}}\left({ }^{s} \boldsymbol{\omega}^{*}\right)=\frac{1}{2}{ }^{s} \dot{\boldsymbol{\omega}}^{* T} \boldsymbol{I} \boldsymbol{\omega}^{*}+\frac{1}{2}{ }^{s} \boldsymbol{\omega}^{* T} \boldsymbol{I}^{s} \dot{\boldsymbol{\omega}}^{*}={ }^{s} \boldsymbol{\omega}^{* T} \boldsymbol{I}^{s} \dot{\boldsymbol{\omega}}^{*}
$$

from which, taking into account that $\boldsymbol{I}^{s} \dot{\boldsymbol{\omega}}^{*}=\boldsymbol{I}^{s} \dot{\boldsymbol{\omega}}$ and Eq. (23), it is possible to derive the following expression:

$$
\dot{E}_{\mathrm{kin}}\left({ }^{s} \boldsymbol{\omega}^{*}\right)=-k^{s} \boldsymbol{\omega}^{* T s} \boldsymbol{B} \times\left({ }^{s} \boldsymbol{\omega}-{ }^{s} \boldsymbol{\omega}_{d}\right) \times{ }^{s} \boldsymbol{B}-{ }^{s} \boldsymbol{\omega}^{* T}\left({ }^{s} \boldsymbol{\omega} \times \boldsymbol{I}^{s} \boldsymbol{\omega}\right)
$$

As ${ }^{s} \omega_{d}$ is parallel to the $z_{s}$ axis, in axisymmetric satellites, the previous equation can be simplified as

$$
\dot{E}_{\mathrm{kin}}\left({ }^{s} \boldsymbol{\omega}^{*}\right)=-k^{s} \boldsymbol{\omega}^{* T s} \boldsymbol{B} \times\left({ }^{s} \boldsymbol{\omega}-{ }^{s} \boldsymbol{\omega}_{d}\right) \times{ }^{s} \boldsymbol{B}
$$

In accordance with expression (4), the previous equation can be rewritten as

$$
\dot{E}_{\mathrm{kin}}\left({ }^{s} \boldsymbol{\omega}^{*}\right)=-k^{s} \boldsymbol{\omega}^{* T} \boldsymbol{S}\left({ }^{s} \boldsymbol{B}\right)^{T} \boldsymbol{S}\left({ }^{s} \boldsymbol{B}\right)^{s} \boldsymbol{\omega}^{*}
$$

Bearing in mind that matrix $\boldsymbol{S}\left({ }^{s} \boldsymbol{B}\right)^{T} \boldsymbol{S}\left({ }^{s} \boldsymbol{B}\right)$ is positive semidefinite [27], the derivative of the Lyapunov candidate function is negative semidefinite if $k>0$. Therefore, according to statement $(21),{ }^{s} \omega^{*}=$ 0 (that is, ${ }^{s} \omega={ }^{s} \omega_{d}$ ) is a stable solution with regard to the law of control chosen. The magnetic field ${ }^{s} \boldsymbol{B}$ depends on ${ }^{s} \boldsymbol{\omega}^{*}$ and the position in the orbit, while ${ }^{s} \omega^{*}$ depends on the dynamic equation of motion. There is no trajectory of the system that makes $\dot{E}_{\mathrm{kin}}\left({ }^{s} \omega^{*}\right)=$ 0 at every moment, except the trivial one ${ }^{s} \omega^{*}=0$. Hence, Krasovskii-LaSalle [28] criteria are met, and as long as Eq. (16) is valid (i.e. $\left\|^{s} \boldsymbol{\omega}-{ }^{s} \boldsymbol{\omega}_{d}\right\| \gg \Omega_{o}$ ), it is possible to state that this solution is not only stable but also asymptotically stable to ${ }^{s} \boldsymbol{\omega}={ }^{s} \boldsymbol{\omega}_{d}$. The 
previous statement entails that the satellite's rotation rate ${ }^{s} \omega$ will tend toward the target value ${ }^{s} \omega_{d}$, save for errors around $\Omega_{o}$ with $\Omega_{o} \ll \omega_{d}$.

For nonaxisymmetric satellites, the stability of the solution ${ }^{s} \omega=$ ${ }^{s} \omega_{d}$ will depend on which of the principal inertia axes around which the satellite is rotating. The gyroscopic coupling term [second term of the right side of Eq. (18)] introduces instability when rotation is produced around the principal inertia axis, the moment of inertia of which has an intermediate value [29]. Therefore, in axisymmetric satellites, the rotation rate will only converge to ${ }^{s} \omega={ }^{s} \omega_{d}$ when the rotation is produced around the principal axis with the highest or lowest moment of inertia.

The orbit's inclination angle affects the present control performance. Controllability characteristics of magnetic controls based on $B$-dot strategy tend to improve with orbit inclination [12], this kind of control not being very efficient for equatorial or near-equatorial orbits (as the magnetic field remains almost constant in magnitude and direction in these orbits). In the present work, only orbits with inclination within the range $i \in[45 \mathrm{deg}, 135 \mathrm{deg}]$ are considered.

The gain value $k$ can be related to the rotation rate correction characteristic time $t_{\text {ang. }}$. The order of magnitude of the satellite's kinetic energy at initial conditions can be estimated with Eq. (22), whereas with Eq. (27), it is possible to establish the order of magnitude of the control subsystem energy dissipation. If all moments of inertia are considered to have the same order of magnitude, $I \sim I_{\|}$, it is then possible to obtain the expression

$$
\dot{E}_{\text {kin }} \simeq-\frac{2 k\|\boldsymbol{B}\|^{2}}{I_{\|}} E_{\text {kin }}
$$

which leads to a first estimation of the characteristic time $t_{\text {ang }}$, needed by the control subsystem to reduce the kinetic energy from the initial value $E_{\text {kin } 0}$ to the final one $E_{\text {end }}$ :

$$
t_{\text {ang }} \sim \frac{\log \left(E_{\text {kin } 0} / E_{\text {end }}\right)}{2 k\|B\|^{2} / I_{\|}}
$$

\section{Attitude Evolution and Rotation Rate Stabilization}

Once the angular rotation rate of the satellite ${ }^{s} \omega$ approaches the target one ${ }^{s} \omega_{d}$,

$$
\left\|{ }^{s} \omega-{ }^{s} \omega_{d}\right\| \sim \Omega_{o}
$$

the second term of the right side of Eq. (16) is no longer negligible. Therefore, the previously neglected torque,

$$
\tau=-k_{0}^{s} A\left[{ }^{0} \dot{B}(t) \times{ }^{0} \boldsymbol{B}(t)\right]
$$

should be taken into account. In the absence of any perturbation, this torque will determine the direction vector of the satellite rotation axis. Furthermore, as explained in this subsection, the satellite tends to align its rotation axis $z_{s}$ with this torque, that is, the direction of vector ${ }^{0} \dot{\boldsymbol{B}}(t) \times{ }^{0} \boldsymbol{B}(t)$.

Both the magnitude and direction of vector ${ }^{0} \dot{\boldsymbol{B}}(t) \times{ }^{0} \boldsymbol{B}(t)$ can be calculated using Eqs. (5) and (11). Two conclusions arise from such calculations. On the one hand, an averaged value of the magnitude of such a torque can be approximated, using Eq. (5), as

$$
\|\tau\| \sim k \frac{9}{2} \Omega_{o} \frac{R_{E}^{6} H_{0}^{2}}{R_{0}^{6}}
$$

On the other hand, the calculations show that the direction of vector ${ }^{0} \dot{\boldsymbol{B}}(t) \times{ }^{0} \boldsymbol{B}(t)$ is affected by the orbit's inclination. As an example, see in Fig. 2 the value of the misalignment angle between the direction of vector ${ }^{0} \dot{\boldsymbol{B}}(t) \times{ }^{0} \boldsymbol{B}(t)$ and the $Z_{0}$ axis $\alpha$, calculated for a sun-synchronous orbit at $700 \mathrm{~km}$ altitude and $i=98 \mathrm{deg}$ inclination. In the figure, it can be appreciated that, for this almost polar orbit, the misalignment has a maximum value of $18 \mathrm{deg}$, a minimum value of zero deg, and an averaged value of $6 \mathrm{deg}$. If these calculations

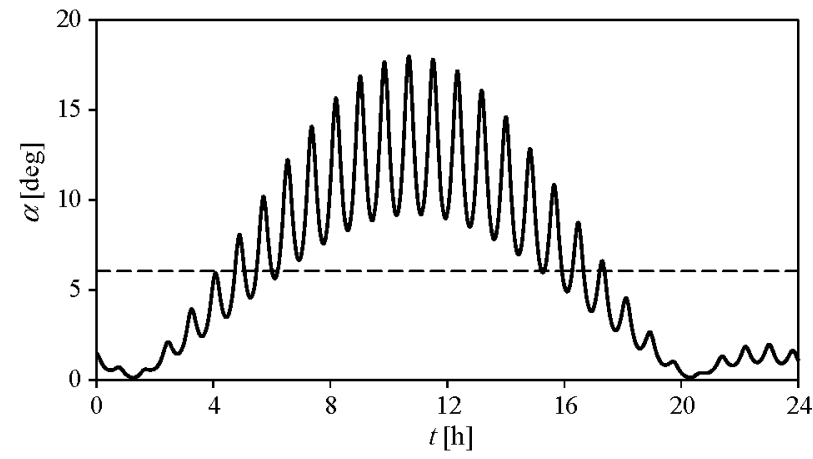

Fig. 2 Misalignment angle $\alpha$ between the direction of the vector of ${ }^{0} \dot{B}(t) \times{ }^{0} B(t)$ and the $Z_{0}$ axis, for a sun-synchronous orbit at $700 \mathrm{~km}$ altitude and $i=98 \mathrm{deg}$ inclination. The averaged value is indicated with a dashed line in the graph.

are repeated for orbits with different inclinations, it is possible to obtain the value of those maximum, minimum, and averaged values as a function of the orbit's inclination; see Fig. 3. In this figure, reduced values of the misalignment angle $\alpha$ are shown for polar orbits; that is, if $i \sim 90 \mathrm{deg}$, the direction of vector ${ }^{0} \dot{\boldsymbol{B}}(t) \times{ }^{0} \boldsymbol{B}(t)$ almost matches the normal vector of the orbit's plane. Besides, it should be also mentioned that the integral of ${ }^{0} \dot{\boldsymbol{B}}(t) \times{ }^{0} \boldsymbol{B}(t)$ during one orbit is, for any inclination, parallel to the direction perpendicular of the orbit.

\section{A. Gyroscopic Explanation of Attitude Evolution}

In this stage, the angular moment of the satellite $\boldsymbol{h}$ is almost parallel to the target velocity ${ }^{s} \omega_{d}$, which is aligned with $z_{s}$. Therefore, the component of the angular moment along the $z_{s}$ axis $h_{z}$ is much greater than the other two components $h_{x}$ and $h_{y}$, and the rotation rate remains fairly constant, as indicated with the stability analysis in the previous subsection. Then, if dissipative effects of the angular rate correction torque are not considered, in this second stage, the satellite dynamics can be approximated to a gyroscope (with the rotation axis in $z_{s}$ ) subjected to a $\tau$ torque.

To demonstrate that the rotation axis of the satellite tends to align with a torque $\tau$, we suppose that this torque has constant direction in inertial axes $F_{0}$. The evolution of the satellite's $z_{s}$ axis with respect to the torque $\tau$ can be explained using Euler angles and supposing $\tau$ parallel to $Z_{0}$ (this condition does not represent any additional hypothesis, as the same equations can be obtained using an inertial reference frame with the $z$ axis parallel to $\tau$ ).

Following a 3-1-3 typical rotation sequence, the first rotation $\phi$ is around the $Z_{0}$ axis, the second rotation $\theta$ is around the $X_{1}$ axis, and the third rotation $\psi$ is around the $Z_{2}$ axis (which coincides with the $z_{\S}$ axis); see Fig. 4. The angular rotation rate is then $\omega=\phi^{0} \hat{\boldsymbol{e}}_{z}+\dot{\theta}^{2} \hat{\boldsymbol{e}}_{x}+\dot{\psi}^{2} \hat{\boldsymbol{e}}_{z}$.

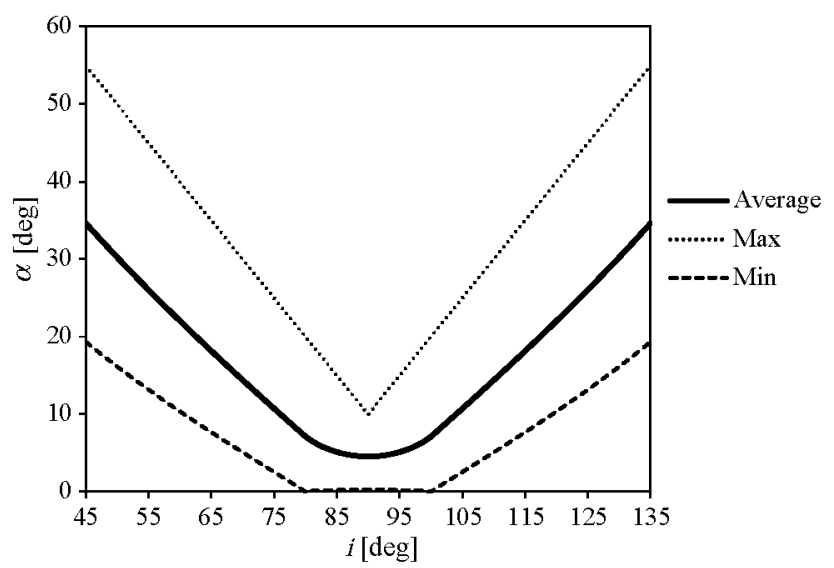

Fig. 3 Maximum, minimum, and averaged values of the misalignment angle $\alpha$ between the direction of the vector of ${ }^{0} \dot{B}(t) \times{ }^{0} B(t)$ and the $Z_{0}$ axis, for orbits at $700 \mathrm{~km}$ altitude, as a function of the inclination, $i$. 


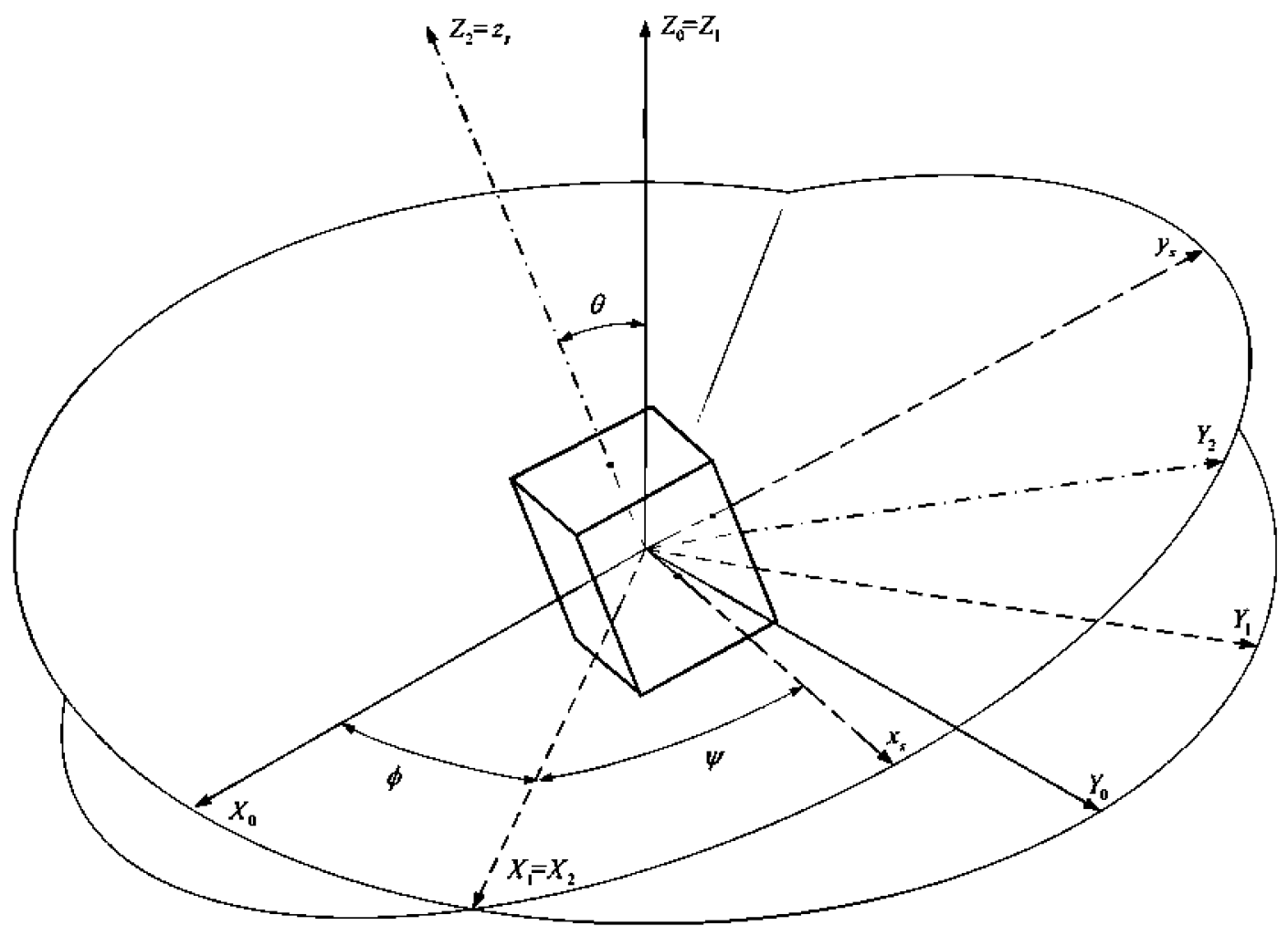

Fig. 4 Sketch of the satellite showing the Euler angles.

The spin axis direction movement can be studied in frame $F_{2}$ (see Fig. 4) using the first $(\theta)$ and second $(\phi)$ Euler angles. Expressing the Euler law in this frame leads to

$$
{ }^{2} \dot{\boldsymbol{h}}+{ }^{2} \Omega_{02} \times{ }^{2} \boldsymbol{h}={ }^{2} \boldsymbol{T}
$$

where the angular moment $\boldsymbol{h}$ can be obtained multiplying the components of the angular rotation rate $\omega$ by the inertia matrix $I$. On the $F_{2}$ frame,

$$
{ }^{2} \boldsymbol{h}=\left[\begin{array}{c}
I_{\perp} \dot{\theta} \\
I_{\perp} \dot{\phi} \sin \theta \\
I_{\|}(\dot{\psi}+\dot{\phi} \cos \theta)
\end{array}\right]
$$

The angular rotation rate $F_{2}$ frame with respect to the inertial $F_{0}$ frame can be expressed as

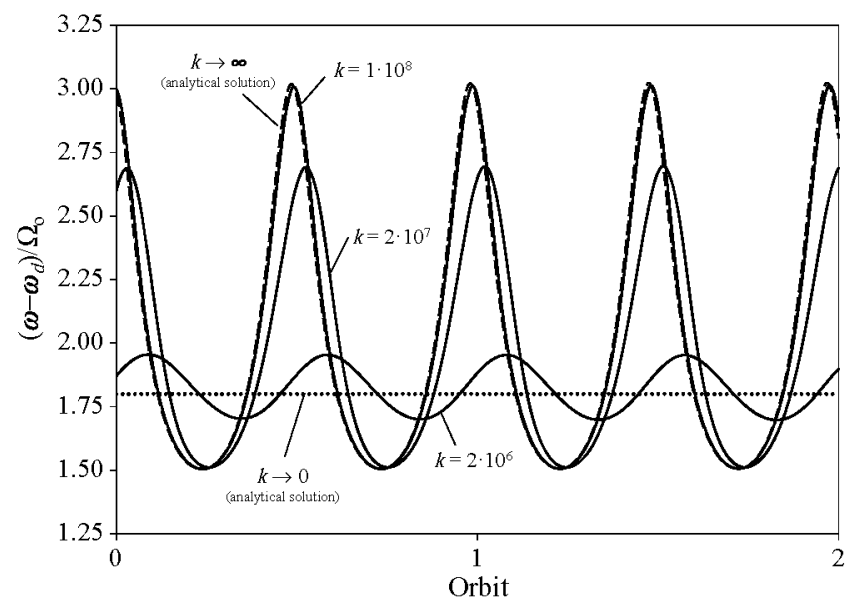

Fig. 5 Nondimensional difference between the stabilization angular velocity and the target rotation rate, $\left({ }^{s} \omega-{ }^{s} \omega_{d}\right) / \Omega_{0}$, plotted along two orbits for a satellite equipped with the proposed control strategy.

$$
{ }^{2} \boldsymbol{\Omega}_{02}=\left[\begin{array}{c}
\dot{\theta} \\
\dot{\phi} \sin \theta \\
\dot{\phi} \cos \theta
\end{array}\right]
$$

Then, substituting the previous expressions in the Euler law, Eq. (33), the equations for each axis are obtained [30],

$I_{\perp}\left(\ddot{\theta}-\cos \theta \sin \theta \dot{\phi}^{2}\right)+I_{\|} \dot{\phi} \sin (\dot{\psi}+\dot{\phi} \cos \theta)=0$

$I_{\perp}(2 \cos \theta \dot{\theta} \dot{\phi}+\sin \theta \ddot{\phi})-I_{\|} \dot{\theta}(\dot{\psi}+\dot{\phi} \cos \theta)=\tau \sin \theta$

$I_{\|} \frac{\mathrm{d}}{\mathrm{d} t}(\dot{\psi}+\dot{\phi} \cos \theta)=0$

where $T_{z}=0$, as it is not possible to apply torque in the rotation axis in gyroscopes. The angular momentum $h_{z}$ around the $Z_{2}$ axis (which coincides with the $z_{s}$ axis; see Fig. 4 ) is

$$
h_{z}=I_{\|} \omega_{z}=I_{\|}(\cos \theta \dot{\phi}+\dot{\psi})
$$

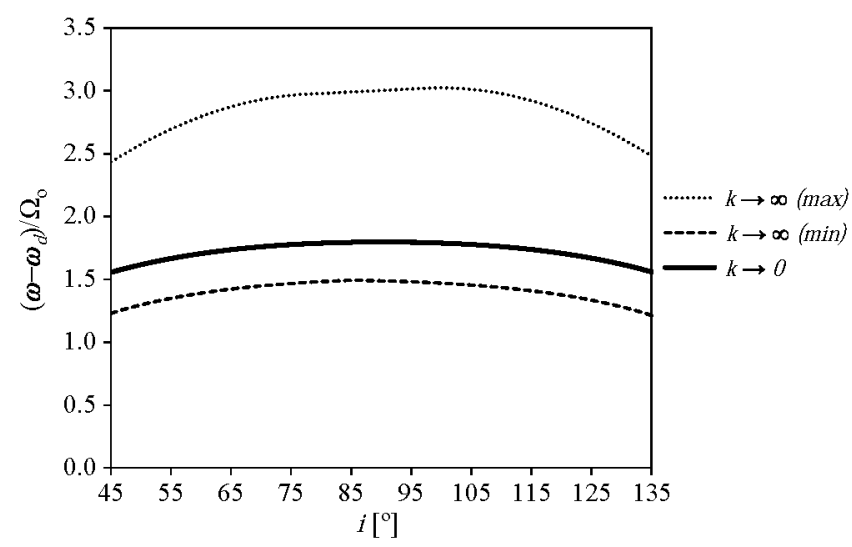

Fig. 6 Maximum and minimum peaks of the nondimensional difference between the stabilization angular velocity and the target rotation rate, $\left({ }^{s} \omega-{ }^{s} \omega_{d}\right) / \Omega_{0}$, as a function of the orbit's inclination. 


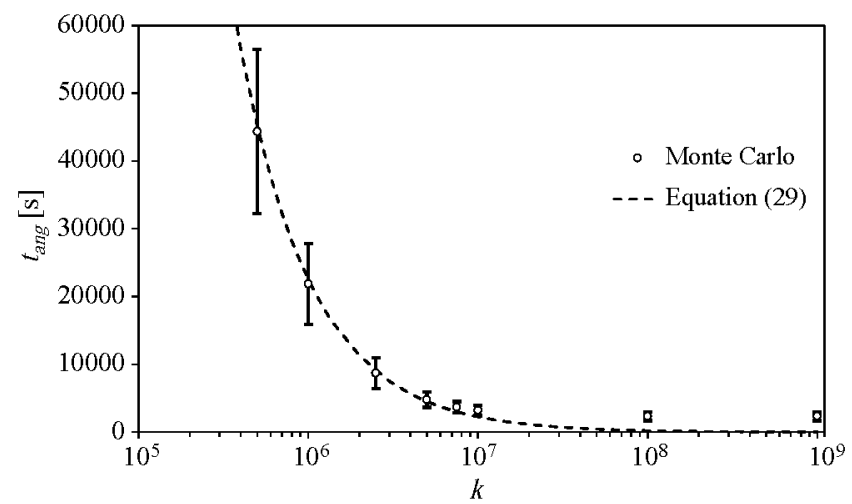

Fig. 7 Results of the first Monte Carlo analysis, carried out to study the characteristic time $t_{\text {ang }}$ needed to stabilize the rotation rate.

Expression (36) can be rewritten as

$$
\begin{aligned}
& I_{\perp}\left(\ddot{\theta}-\cos \theta \sin \theta \dot{\phi}^{2}\right)+h_{z} \sin \theta \dot{\phi}=0 \\
& I_{\perp}(2 \cos \theta \dot{\theta} \dot{\phi}+\sin \theta \ddot{\phi})-h_{z} \dot{\theta}=\tau \sin \theta \\
& \dot{h}_{z}=0
\end{aligned}
$$

Bearing in mind that $\dot{\psi} \gg \dot{\phi}$ and $\dot{\theta}$ as ${ }^{s} h_{z} \gg{ }^{s} h x$ and ${ }^{s} h_{y}$, the second equation on Eq. (38) can be reduced to the following one:

$$
h_{z} \dot{\theta} \simeq-\tau \sin \theta
$$

Hence, it is possible to deduce the behavior of rotation $\theta$ between the $F_{2}$ and $F_{0}$ frames during this alignment phase:

$$
\dot{\theta} \simeq-\frac{\tau}{h_{z}} \sin \theta \simeq-\frac{\tau}{I_{\|} \dot{\psi}} \sin \theta=-\frac{\tau}{I_{\|} \omega_{d}} \sin \theta
$$

That is, the variation of rotation $\theta$ is defined by the equation

$$
\theta(t)=2 \tan ^{-1}\left[\tan \left(\frac{\theta_{0}}{2}\right) \mathrm{e}^{-\left(\tau / I_{\mid} \omega_{d}\right) t}\right]
$$

which implies that rotation $\theta$ tends asymptotically to $\theta=0$; this means that the $z_{s}$ and $Z_{0}$ axes tend to be aligned (i.e., $z_{s}$ tends to be aligned with $\tau$ ).

In the present case, $\tau$ does not have a constant inertial direction; it varies periodically along the orbit. For this reason [and after a stabilization period that can be estimated according to Eq. (41)], the rotation axis direction $z_{S}$ will remain between the one from the torque $\boldsymbol{\tau}$ [parallel to ${ }^{0} \dot{\boldsymbol{B}}(t) \times{ }^{0} \boldsymbol{B}(t)$ ] at each moment and the average value of this direction along one orbit $\left(Z_{0}\right)$. The rotation axis direction $z_{s}$ will approach the first or the second option depending on the gain $k$ and therefore on the subsystem's reaction speed.

\section{B. Rotation Rate Stabilization}

As stated in the previous section, the satellite's rotation axis $z_{s}$ tends to align with the torque $\tau$, which happens to have the same direction of vector ${ }^{0} \dot{\boldsymbol{B}}(t) \times{ }^{0} \boldsymbol{B}(t)$. Then, if $z_{s}$ is supposed to be aligned with ${ }^{0} \dot{\boldsymbol{B}}(t) \times{ }^{0} \boldsymbol{B}(t)$, the satellite dynamic Eq. (16) becomes

$$
\left.\boldsymbol{I}^{s} \dot{\boldsymbol{\omega}}=-k^{s} \boldsymbol{B} \times\left({ }^{s} \boldsymbol{\omega}\right)-{ }^{s} \boldsymbol{\omega}_{d}\right) \times{ }^{s} \boldsymbol{B}-k\left\|\left({ }^{0} \dot{\boldsymbol{B}}(t) \times{ }^{0} \boldsymbol{B}(t)\right)\right\| \|^{s} \hat{\boldsymbol{e}}_{z}-{ }^{s} \boldsymbol{\omega} \times \boldsymbol{I}^{s} \boldsymbol{\omega}
$$

Which, introducing the change of variable
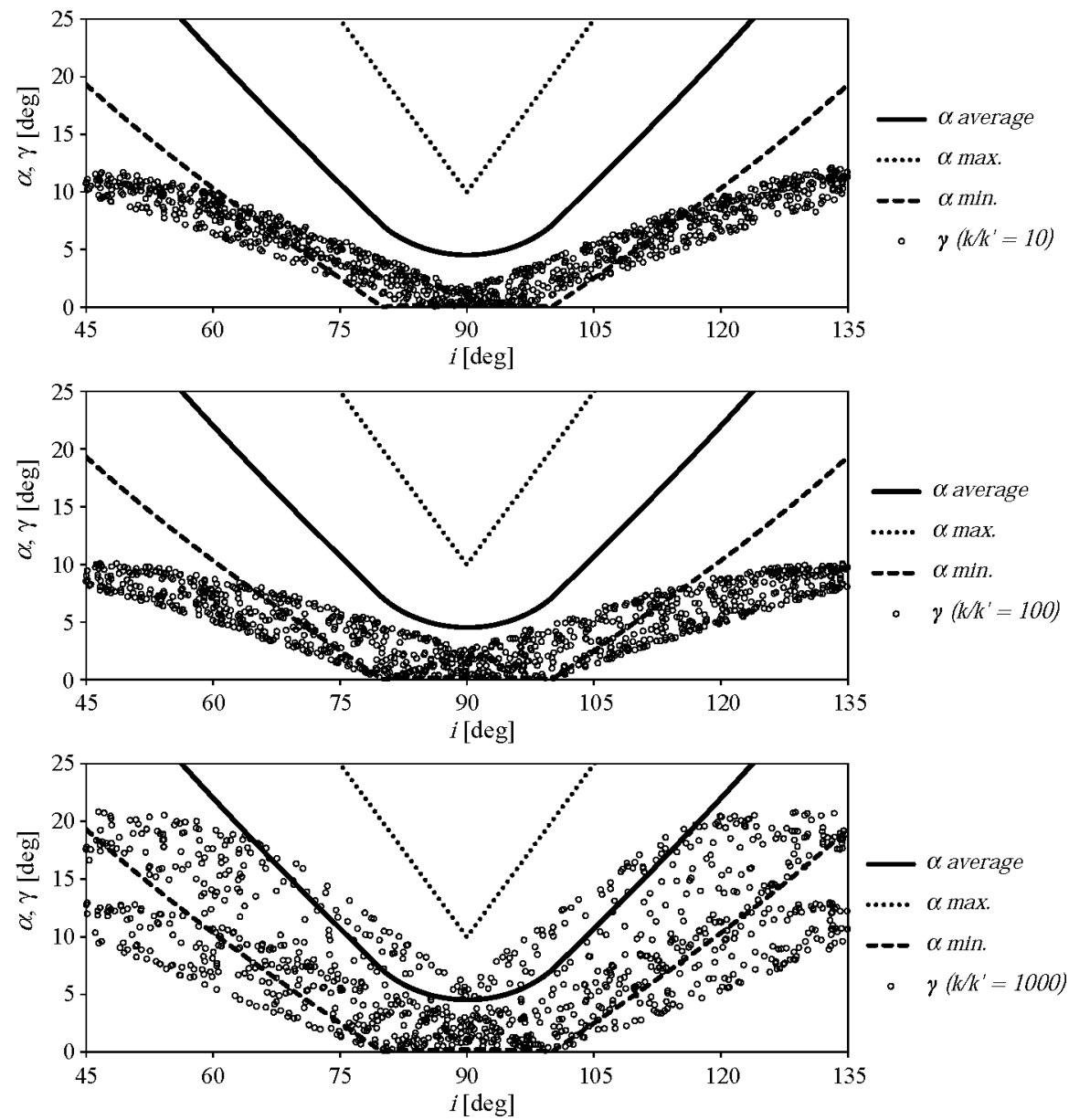

Fig. 8 Results of the second Monte Carlo analysis, carried out to study the situation once the rotation rate is stabilized for control gain constants $k=1 \cdot 10^{7}, 1 \cdot 10^{8}$, and $1 \cdot 10^{9}\left(k^{\prime}=1 \cdot 10^{6}\right.$ in the graphs' legends $)$. 


$$
{ }^{s} \boldsymbol{\omega}_{d}^{*}(t)=\left(\omega_{d}+\frac{\left\|^{0} \dot{\boldsymbol{B}}(t) \times \boldsymbol{B}(t)\right\|}{\|\boldsymbol{B}(t)\|^{2}}\right){ }^{s} \hat{\boldsymbol{e}}_{z}
$$

turns into an equation quite similar to the one from expression (16):

$$
\boldsymbol{I}^{s} \dot{\boldsymbol{\omega}}=-k^{s} \boldsymbol{B} \times\left({ }^{s} \boldsymbol{\omega}-{ }^{s} \boldsymbol{\omega}_{d}^{*}\right) \times{ }^{s} \boldsymbol{B}-{ }^{s} \boldsymbol{\omega} \times \boldsymbol{I}^{s} \boldsymbol{\omega}
$$

However, it should be emphasized that in the previous expression the new variable ${ }^{s} \omega_{d}^{*}$ depends on time $t$ and periodically varies as the satellite travels along the orbit. Because of this variation, the stationary solution of Eq. (44) depends on the control constant $k$. For large values of $k, k \gg I_{\|} \Omega_{o} /\|\boldsymbol{B}\|^{2}$, the variable $\omega_{d}^{*}$ can be considered constant in each position of the orbit, as the characteristic time of the control reaction is small when compared to the orbital period. As a result, Eq. (44) can be solved considering $\omega_{d}^{*}$ constant in every position of the orbit. On the other hand, for small values of $k$, $k \ll I_{\|} \Omega_{o} /\|B\|^{2}$, the characteristic time of the control reaction is large when compared to the orbital period. Consequently, both $\left\|{ }^{0} \boldsymbol{B}(t) \times{ }^{0} \boldsymbol{B}(t)\right\|$ and $\|\boldsymbol{B}(t)\|^{2}$ can be, respectively, approximated by their average values. For intermediate values of $k$, the stationary solution should be somewhere between both extreme solutions $(k \rightarrow \infty$ and $k \rightarrow 0$ ). See in Fig. 5 an example of the mentioned behaviors. In the figure, the nondimensional difference between the stabilization angular velocity and the target rotation rate, $\left({ }^{s} \omega-\right.$ $\left.{ }^{s} \omega_{d}\right) / \Omega_{0}$, for sun-synchronous orbits at $700 \mathrm{~km}$ altitude and $i=$ $98 \mathrm{deg}$ inclination, with different values of the gain constant $k$, is plotted as a function of time during two orbital periods. The aforementioned extreme solutions are also plotted. In the graph, it can be appreciated that the error in the angular velocity is limited to a value between 1.5 and 3 times the orbital rotation rate $\Omega_{o}$, these limits being the maximum and minimum values of $\left\|{ }^{0} \dot{\boldsymbol{B}}(t) \times{ }^{0} \boldsymbol{B}(t)\right\| /\|\boldsymbol{B}(t)\|^{2}$ along the orbit. For lower values of the control gain constant, the solution approaches the averaged value represented by $\int_{0}^{T} \|^{0} \dot{\boldsymbol{B}}(t) \times$ ${ }^{0} \boldsymbol{B}(t)\left\|/ \int_{0}^{T}\right\| \boldsymbol{B}(t) \|^{2}$, which represents the value corresponding to the limit $k \rightarrow 0$. These calculations have been repeated for orbits with different inclinations within the bracket [ $45 \mathrm{deg}, 135 \mathrm{deg}$ ], the results being included in Fig. 6. In this graph, the maximum and minimum values of the bracket, in which the nondimensional difference between the stabilization angular velocity and the target rotation rate, $\left({ }^{s} \omega-{ }^{s} \omega_{d}\right) / \Omega_{0}$, oscillates, are plotted as a function of the orbit's inclination $i$. Also, the values corresponding to the limit $k \rightarrow 0$ at each inclination are plotted in the graph.

\section{Results and Discussion}

\section{A. Monte Carlo Simulations}

A Monte Carlo simulation was carried out to confirm the conclusions arising from the previous subsections and to validate the aforementioned prediction of the characteristic time $t_{\text {ang }}$ [Eq. (29)]. Eight control gain values $k=5 \cdot 10^{5}, 1 \cdot 10^{6}, 2.5 \cdot 10^{6}, 5 \cdot 10^{6}$, $7.5 \cdot 10^{6}, 1 \cdot 10^{7}, 1 \cdot 10^{8}$, and $1 \cdot 10^{9}$ were considered. For each one of them, 1000 cases were analyzed, randomly varying the values of these 12 parameters:

1) Parameters 1-3: The three values of the moments of inertia, with $I_{x}, I_{y} \in[2,4] \mathrm{kg} \cdot \mathrm{m}^{2}$ and $I_{z}$ being either 2 or $4 \mathrm{~kg} \cdot \mathrm{m}^{2}$ (which, as explained, ensures stability of the rotation around the $z$ axis).

2) Parameters 4-6: The three values of the unit vector that characterizes the initial attitude of the satellite.
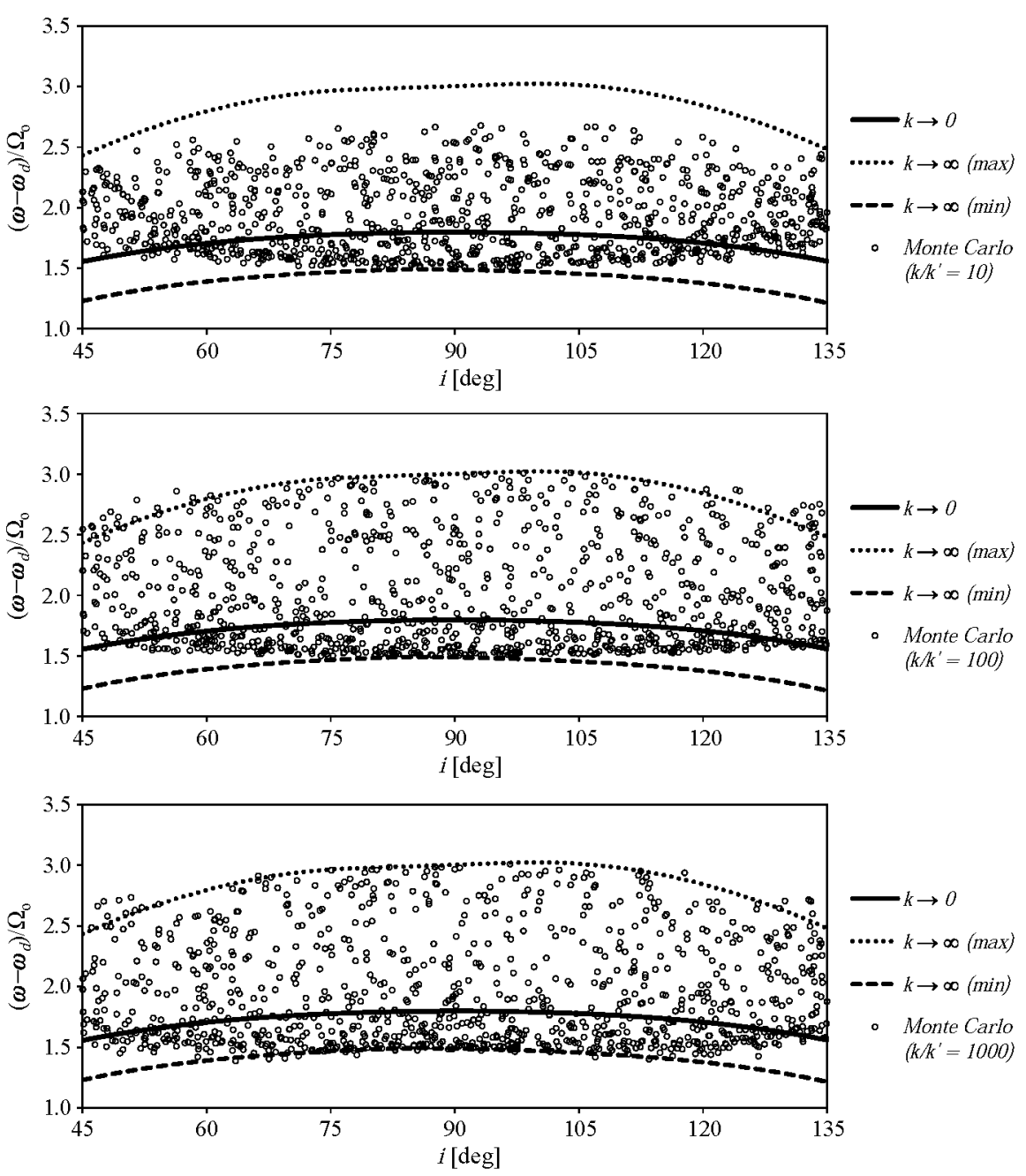

Fig. 9 Results of the second Monte Carlo analysis, carried out to study the situation once the rotation rate is stabilized for control gain constants $k=1 \cdot 10^{7}, 1 \cdot 10^{8}$, and $1 \cdot 10^{9}\left(k^{\prime}=1 \cdot 10^{6}\right.$ in the graphs' legends $)$. 
3) Parameter 7: The initial rotation angle between the $F_{E}$ frame and $F_{i}$ frame, with $\Theta_{T 0} \in[0,2 \pi]$.

4) Parameter 8: The initial value of the latitude position in the considered circular orbit, with $u_{0} \in[0,2 \pi]$.

5) Parameter 9: The inclination of the orbit, with $i \in[45 \mathrm{deg}, 135 \mathrm{deg}]$.

6) Parameters 10-12: The three components of the satellite's rotation rate $\omega_{x 0}, \omega_{y 0}$, and $\omega_{z 0}$. The initial value of the rotation rate was chosen according to the following condition:

$$
E_{\mathrm{kin} 0}=I_{x} \omega_{x 0}^{2}+I_{y} \omega_{y 0}^{2}+I_{z}\left(\omega_{z 0}-\omega_{d}\right)^{2}=0.36
$$

In all cases, the target rotation rate was ${ }^{s} \omega_{d}=0.1^{s} \hat{\boldsymbol{e}}_{z} \mathrm{rad} / \mathrm{s}$, the maximum magnetic torque level being $\pm 15 \mathrm{~A} \mathrm{~m}^{2}$ in each axis. The characteristic time needed to reduce the kinetic energy to $E_{\text {end }}=$ $10\left(I_{x}+I_{y}+I_{z}\right) \Omega_{o}^{2}$ was obtained in every case, this limit being taken as the end of the rotation rate correction (the order of magnitude of the satellite's rotation rate once at the end of this stage is around $\Omega_{o}$; see Sec. V). The average value (with its associated standard deviation bars) is plotted in Fig. 7, for each one of the selected control gain values, together with the predicted values of the characteristic time $t_{\text {ang }}$. The results from the figure show quite efficient rotation rate correction, together with the accuracy of the proposed estimation for the characteristic time. Besides, it should also be pointed out that the results show no improvement on the characteristic time for values beyond $k=1 \cdot 10^{8}$, due to the limitation on the magnetic torque acting on the satellite (limited to $\pm 15 \mathrm{~A} \mathrm{~m}^{2}$ in each axis).

A second Monte Carlo analysis was done, to study the satellite's attitude once the rotation rate was stabilized into a periodical state (see Sec. V). Three control gain constants $k=1 \cdot 10^{7}, 1 \cdot 10^{8}$, and
$1 \cdot 10^{9}$ were selected, 1000 cases being simulated for each one of them, with different initial conditions (see simulation parameters stated at the previous subsection). The averaged value (calculated during one orbit, once the rotation rate was stabilized) of the misalignment angle $\gamma$ between the direction of the satellite's rotation axis $z_{s}$ and the normal direction to the orbit's plane $Z_{0}$ is shown in Fig. 8 as a function of the orbit's inclination, for all the simulations carried out. As this misalignment follows the misalignment angle $\alpha$ between ${ }^{0} \dot{\boldsymbol{B}}(t) \times{ }^{0} \boldsymbol{B}(t)$ and the $Z_{0}$ axis (see Sec. V), the maximum, minimum, and averaged values of this angle have also been included in the graphs of the figure. The dispersion observed in the data is caused by the different angles of the orbit's plane in relation to the Earth magnetic dipole. For larger values of the control gain constant $k$, the dispersion of the results increases as the satellite's $z$-axis vector $z_{s}$ is more capable of following the direction changes of vector ${ }^{0} \dot{\boldsymbol{B}}(t) \times{ }^{0} \boldsymbol{B}(t)$ (that is, for larger values of $k, \gamma$ tends to increase the oscillation between the maximum and the minimum values of $\alpha$ on one orbit; see also Figs. 2 and 3). Finally, it should be mentioned that the highest misalignment between the satellite's rotation axis $z_{s}$ and the orbit's plane normal direction $Z_{0}$ is significantly reduced $(\gamma<5 \mathrm{deg})$, for high-inclination orbits $(i \sim 90 \mathrm{deg})$ and control gain values up to $k=1 \cdot 10^{8}$.

Besides, the nondimensional difference between the stabilization angular velocity and the target rotation rate, $\left({ }^{s} \omega-{ }^{s} \omega_{d}\right) / \Omega_{0}$, was also studied using this Monte Carlo analysis. In Fig. 9, the results corresponding to the value of the aforementioned difference between the stabilization angular velocity and the target rotation rate at the end of all simulations are shown. As expected, larger values of the control gain constant result in a greater dispersion of the results between the limits derived from the analytical solution $k \rightarrow \infty$ (see also Figs. 5 and 6 from Sec. V).
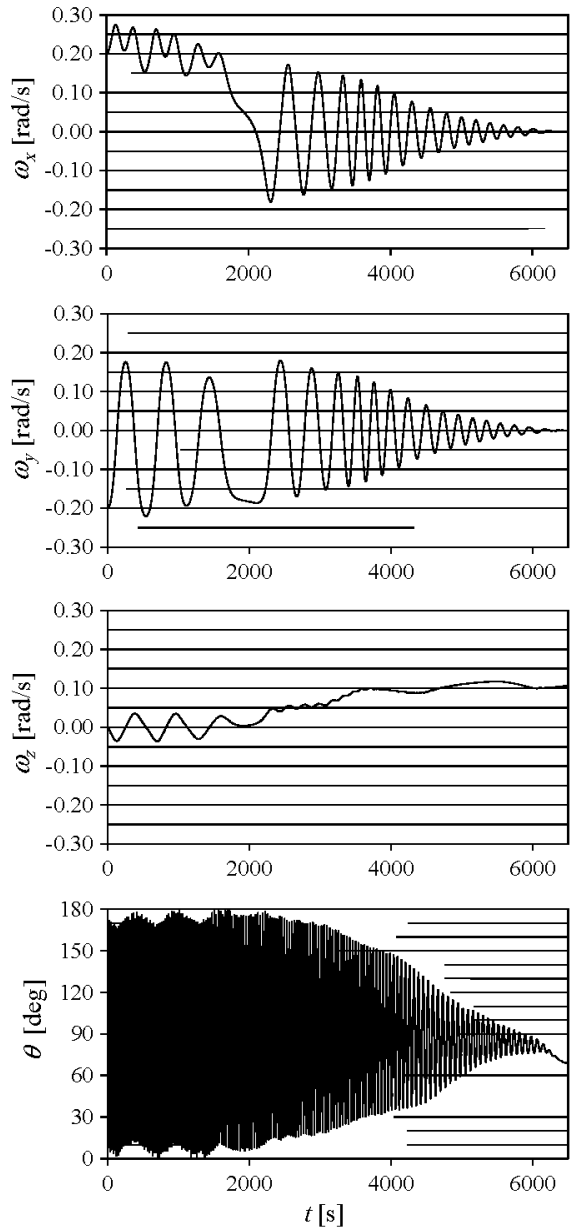
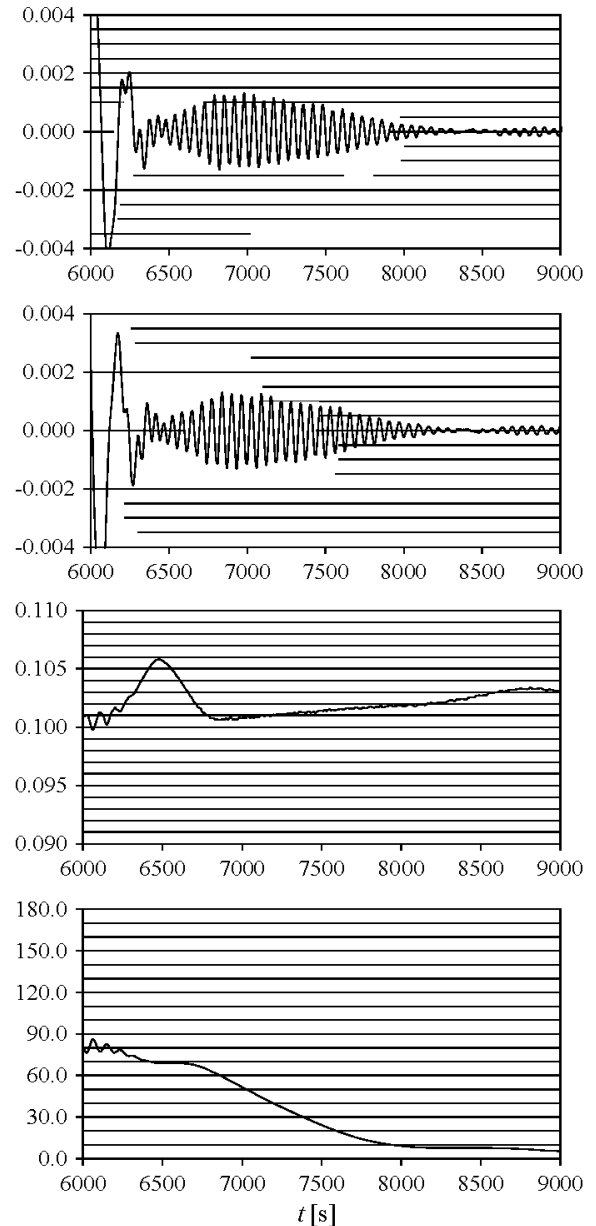

$t[\mathrm{~s}]$
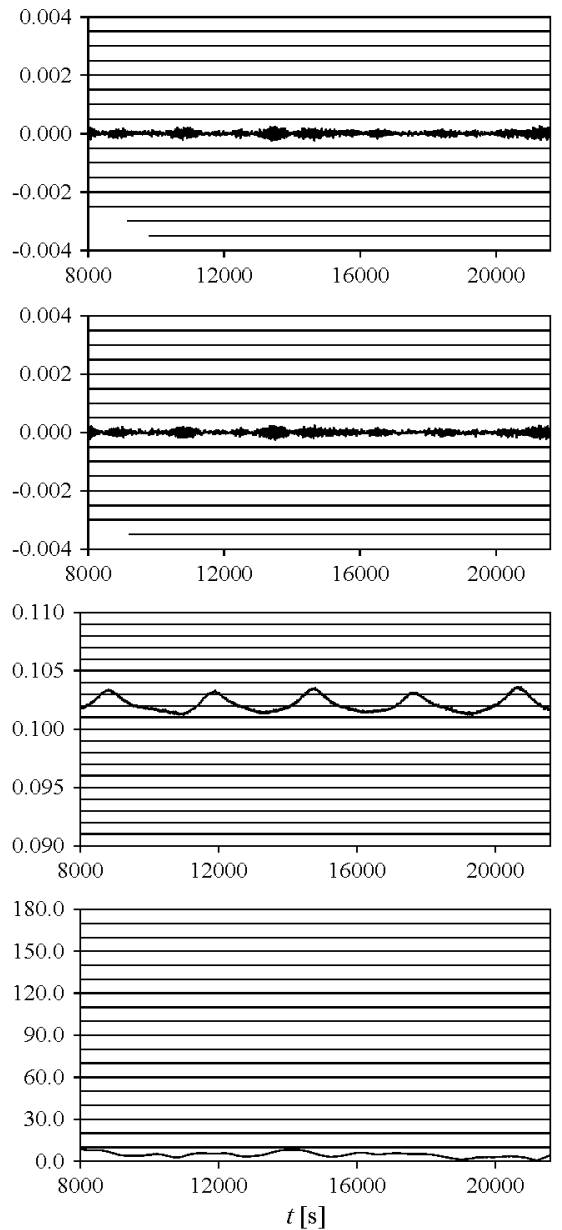

Fig. 10 Evolution over time $t$ of angular velocity components and angle of misalignment $\theta$ for UPMSat-2. Initial conditions: $[\phi, \theta, \psi]=$ $[0 \mathrm{deg}, 30 \mathrm{deg}, 60 \mathrm{deg}]$ and ${ }^{s} \omega_{0}=[0.2,-0.2,0]$. 


\section{B. UPMSat-2}

In the previous sections, the theoretical basics of the proposed control law have been shown, the viability being demonstrated in the absence of any perturbation or error. However, several factors can affect the attitude control performance and consequently should be taken into account in the formulas derived from the ideal satellite dynamics, i.e., Eq. (16). On the one hand, orbit and environmental conditions, like geomagnetic field or perturbations (gravitational perturbations, sun radiation, etc.), have been simplified or neglected in the ideal formulation in previous sections and will be slightly different during spacecraft operation. On the other hand, the control subsystem of a spacecraft is affected by some possible limitations within its operation, such as the discrete-operation mode of the control, delay in signal reception and data processing, sequential operation of magnetic field measurements and magnetic actuation in order to avoid interferences, magnetorquers characteristic time and maximum torque, etc. Despite all these effects, most of them not taken into account in the previous sections, the algorithm developed in the present work should be able to carry out an effective attitude control of the spacecraft.

To check its effectiveness, the proposed attitude control algorithm was implemented in the MATLAB®-SIMULINK UPMSat-2 mission simulator along with the dynamics of the satellite. This simulator integrates different modules, which characterize the UPMSat-2 behavior. Among them it can be pointed out the following ones:

1) The inertia matrix of UPMSat-2.

2) An accurate simulation of the expected orbit for UPMSat-2

3) A detailed geomagnetic field model based on National Oceanic and Atmospheric Administration 2010-2015 World Magnetic Model [26].

4) Several models of the major perturbations along the orbit (e.g., torques due to solar and aerodynamic effects).
5) The alternated operation of magnetometers and magnetorquers, to avoid interaction between them.

6) A model of the magnetorquers performance, which includes the response characteristic time and maximum magnetic torque.

7) A model of the magnetometers performance, including $2 \mathrm{nT}$ (rms) noise and around $25 \mathrm{nT}$ accuracy error.

The performance during $6 \mathrm{~h}$ of the UPMSat- 2 was simulated for nine different initial conditions, three attitudes $[\phi, \theta, \psi]=$ [0 deg, $30 \mathrm{deg}, 60 \mathrm{deg}$ ], [60 deg, $0 \mathrm{deg}, 30 \mathrm{deg}$ ], and [30 deg, $60 \mathrm{deg}$, $0 \mathrm{deg}]$ combined with three different initial rotation rates ${ }^{s} \omega_{0}=[0.2,-0.2,0],[0.2,0,-0.2]$, and $[0,0.2,0.2] \mathrm{rad} / \mathrm{s}$. The target rotation and the control gain were, respectively, ${ }^{s} \omega_{d}=0.1^{s} \hat{\boldsymbol{e}}_{z}$ and $k=2 \cdot 10^{8}$, in every case. Taking into account the maximum dipole that can be supplied by the magnetorquers when activated, $m=15 \mathrm{~A} \mathrm{~m}^{2}$, the gain was set to obtain maximum amplification without saturation during the second phase. With this procedure, the duration of the second stage was minimized, and reduced power consumption was also ensured once the stabilized attitude was reached. Besides, it is also fair to say that magnetorquers reached saturation within the first control stage, as they were unable to produce the required torque. Nevertheless, this saturation of the magnetorquers does not represent any problem in that dissipative first stage.

Three of these simulations are presented in Figs. 10-12. In each one, results are shown in three different consecutive and partially overlapped time sections, with different time scales, in which each one of the different control stages described in the previous section can be clearly appreciated. In a first stage of the attitude control, angle $\theta$ varies uncontrollably, whereas rotation speed components ${ }^{s} \omega_{x}$ and ${ }^{s} \omega_{y}$ tend to zero, and the component ${ }^{s} \omega_{z}$ approaches the target value. This rotation rate correction stage takes for all the cases between 3500 and $6500 \mathrm{~s}$ (see left graphs in Figs. 10-12) its value depending on the initial kinetic energy.
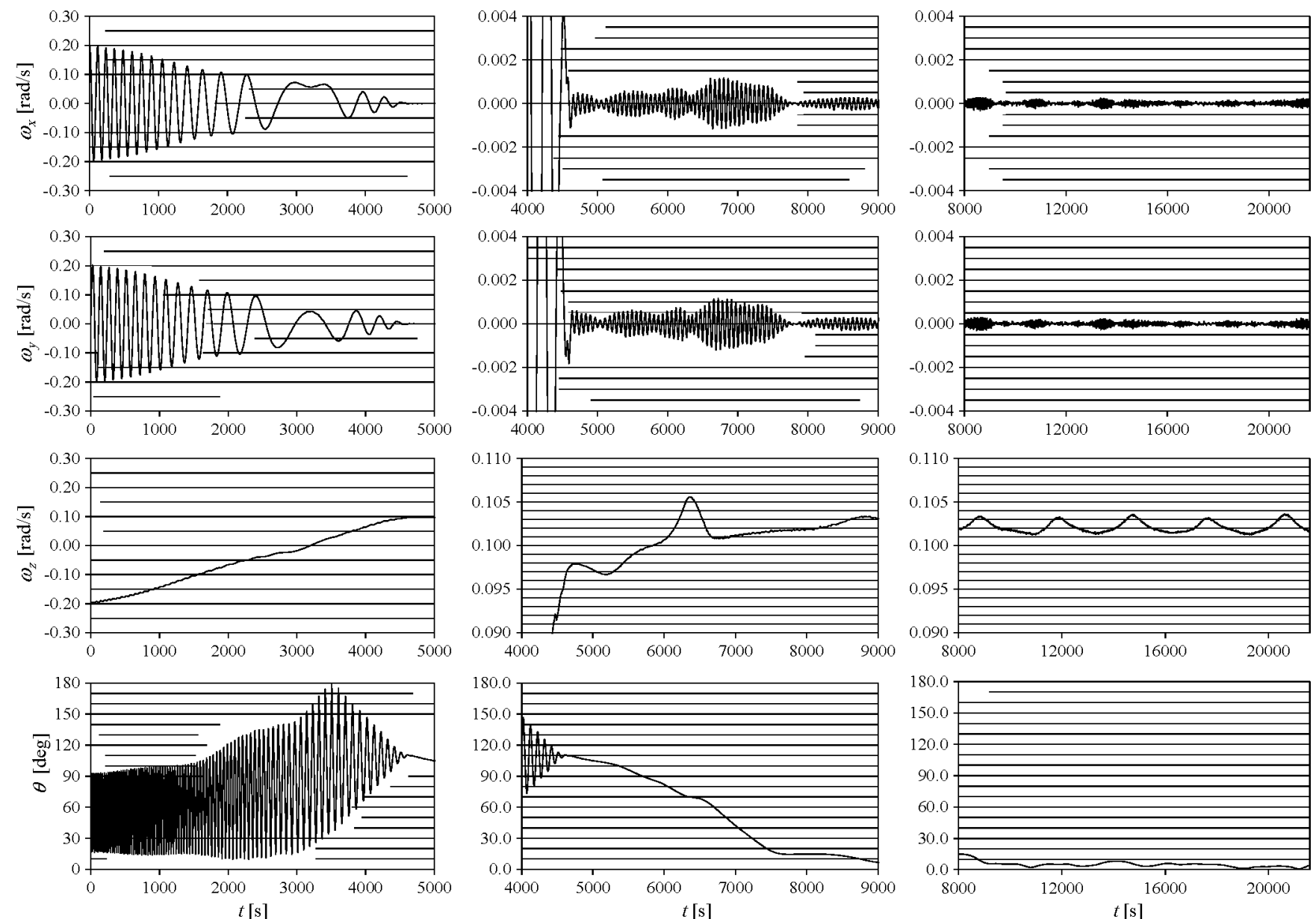

Fig. 11 Evolution over time $t$ of angular velocity components and angle of misalignment $\theta$ for UPMSat-2. Initial conditions: $[\phi, \theta, \psi]=$ [60 deg, $0 \mathrm{deg}, 30 \mathrm{deg}]$ and $^{s} \omega_{0}=[0.2,0,-0.2]$. 

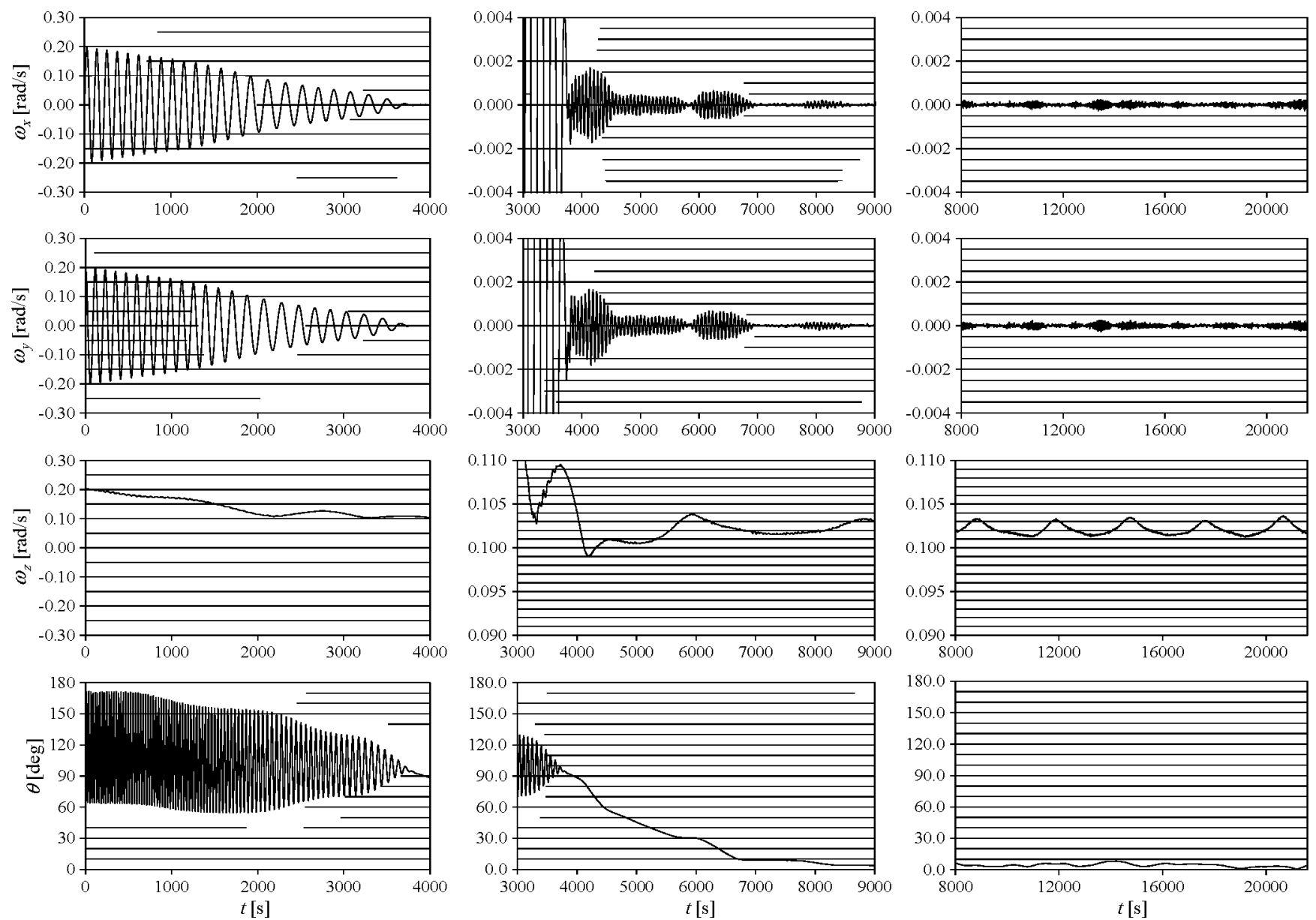

Fig. 12 Evolution over time $t$ of angular velocity components and angle of misalignment $\theta$ for UPMSat-2. Initial conditions: $[\phi, \theta, \psi]=$ $[30 \mathrm{deg}, 60 \mathrm{deg}, 0 \mathrm{deg}]$ and ${ }^{s} \omega_{0}=[0,0.2,0.2]$.

The second stage, regarding the attitude correction process that aligns the rotation axis with the normal vector of the orbit's plane, can be observed in the middle graphs of Figs. 10-12. As mentioned before, in this second stage, the rotation rate was stabilized, and the angle $\theta$ between the satellite rotation axis and the normal vector to the orbit's plane gradually turned to zero. This attitude correction stage took for all the cases between 1500 and $4500 \mathrm{~s}$ (see the central graphs in Figs. 10-12).

The stabilized situation can be appreciated in the right graphs of Figs. 10-12. The attitude of the satellite has a periodical behavior, which is significantly similar in all cases analyzed. Therefore, it can be assumed that the influence of the initial conditions disappears once the satellite has completed the second correction stage. Rotation rate components ${ }^{s} \omega_{x}$ and ${ }^{s} \omega_{y}$ are negligible, whereas ${ }^{s} \omega_{z}$ is within the limits stated in Sec. V. The misalignment (expressed in terms of the $\theta$ angle) is reduced up to $4.5 \mathrm{deg}$ (on average) in all cases.

According to the results from the simulator, it can be assumed that the control algorithm works properly and it is reliable for the UPMSat-2 mission. Furthermore, results indicate good correlation with the analytical approach to the control algorithm, which can be split into three different stages as explained in the previous section.

\section{Conclusions}

In the present work, a new type of magnetic control applied to lowEarth-orbit satellites is described. The proposed control law is able to set the satellite rotation speed around its maximum or minimum inertia principal axis. Besides, the proposed control law favors the alignment of this axis with the normal direction to the orbital plane for high-inclination orbits.

The proposed control algorithm is simple since 1) only magnetorquers are required as actuators, 2) only magnetometers are re- quired as sensors, 3) it does not need an in-orbit Earth magnetic field model, 4) it does not need to be externally activated with information about the orbital characteristics, and 5) it allows automatic reset after a total shutdown of attitude control subsystem.

The theoretical viability of the control law has been checked through Monte Carlo analysis of orbits with inclinations in the bracket [45 deg, $135 \mathrm{deg}$ ]. The results indicate low error levels regarding both the rotation rate correction and the misalignment with target attitude, for high-inclination orbits. The accuracy level of the satellite's rotation rate correction is around the orbital rotation rate, whereas the accuracy in relation to the target attitude is lower than $5 \mathrm{deg}$, for such kinds of orbits.

The proposed algorithm has been tested in the UPMSat-2 simulator. The results showed a very good agreement with all the analytical approaches developed in the present work.

Finally, is noteworthy to mention that, in terms of power production, the proposed attitude (on the principal axis perpendicular to the orbit plane and the satellite rotating around it with a controlled rate) is very suitable for the UPMSat-2 mission, as it allows a higher area of the panels pointing toward the sun when compared to other studied attitudes. Besides, the proposed attitude showed significant improvements, when compared to others, in terms of thermal control, as the satellite angular rotation rate can be selected to achieve a higher temperature homogenization of the satellite

\section{Acknowledgments}

The authors are indebted to Ángel Sanz Andrés for his help and encouraging support regarding the present work. They are indebted to the Instituto Universitario de Microgravedad "Ignacio Da Riva" (IDR/UPM) staff, and especially the colleagues also involved in the UPMSat-2 project for their friendly support. The authors are grateful 
to the library staff at the Escuela de Ingeniería Aeronáutica y del Espacio of the Universidad Politécnica de Madrid for their constant help in providing technical literature and document review. Finally, the authors are also truly grateful to David Carrión and Brian Elder for their kind help on improving the style of the text.

\section{References}

[1] White, J., and Shigemoto, F., "Satellite Attitude Control Utilizing the Earth's Magnetic Field," NASA TN-D-1068, 1961, p. A-474.

[2] De Ruiter, A., "A Fault-Tolerant Magnetic Spin Stabilizing Controller for the JC2Sat-FF Mission," Acta Astronautica, Vol. 68, Nos. 1-2, Jan. 2011, pp. 160-171. doi:10.1016/j.actaastro.2010.07.012

[3] Findlay, E. J., De Ruiter, A., Forbes, J. R., Liu, H. H. T., Damaren, C. J., and Lee, J., "Magnetic Attitude Control of a Flexible Satellite," Journal of Guidance, Control, and Dynamics, Vol. 36, No. 5, Sept. 2013, pp. 1522-1527. doi: $10.2514 / 1.57300$

[4] Ovchinnikov, M. Y., Ilyin, A. A., Kupriynova, N. V., Penkov, V. I., and Selivanov, A. S., "Attitude Dynamics of the First Russian Nanosatellite TNS-0," Acta Astronautica, Vol. 61, Nos. 1-6, June 2007, pp. 277-285. doi:10.1016/j. actaastro.2007.01.006

[5] Coverstone-Carroll, V., "Detumbling and Reorienting Underactuated Rigid Spacecraft," Journal of Guidance, Control, and Dynamics, Vol. 19, No. 3, 1996, pp. 708-710. doi: 10.2514/3.21680

[6] Farrahi, A., and Sanz-Andrés, A., "Efficiency of Hysteresis Rods in Small Spacecraft Attitude Stabilization," Scientific World Journal, Vol. 2013, Jan. 2013, pp. 1-17. doi: $10.1155 / 2013 / 459573$

[7] Stras, L., Kekez, D. D., Wells, G. J., Jeans, T., Zee, R. E., Pranajaya, F., and Foisy, D., "The Design and Operation of the Canadian Advanced Nanospace eXperiment (CanX-1)," Proceedings of the Radio Amateur Satellite Corporation, North America (AMSAT-NA), 21st Space Symposium, Toronto, Ontario, Canada, 2003, pp. 150-160.

[8] Mohammed, A. M., Benyettou, M., Sweeting, M. N., and Cooksley, J. R., "Initial Attitude Acquisition Result of the Alsat-1 First Algerian Microsatellite in Orbit," Proceedings of IEEE Networking, Sensing and Control (ICNSC2005), IEEE Publ., Piscataway, NJ, 2005, pp. 566-571. doi:10.1109/ICNSC.2005.1461252

[9] Bhat, S. P., and Dham, A. S., "Spacecraft Attitude Under Magnetic Actuation," 42nd Institute of Electrical and Electronics Engineers Conference on Decision and Control, IEEE Publ., Piscataway, NJ, 2003, pp. 2383-2388.

[10] Wisniewski, R., and Stoustrup, J., "Periodic H2 Synthesis for Spacecraft Attitude Control with Magnetorquers," Journal of Guidance, Control, and Dynamics, Vol. 27, No. 5, 2004, pp. 874881 . doi: $10.2514 / 1.10457$

[11] Lovera, M., and De Marchi, E., "Periodic Attitude Control Techniques for Small Satellites with Magnetic Actuators," IEEE Transactions on Control Systems Technology, Vol. 10, No. 1, 2002, pp. 90-95. doi: $10.1109 / 87.974341$

[12] Silani, E., and Lovera, M., "Magnetic Spacecraft Attitude Control: A Survey and Some New Results," Control Engineering Practice, Vol. 13, No. 3, March 2005, pp. 357-371. doi:10.1016/j.conengprac.2003.12.017

[13] Ahmed, S., and Kerrigan, E. C., "Suboptimal Predictive Control for Satellite Detumbling," Journal of Guidance, Control, and Dynamics, Vol. 37, No. 3, May 2014, pp. 850-859. doi: $10.2514 / 1.61367$
[14] Flatley, T. W., Morgenstern, W., Reth, A., and Bauer, F., "A B-Dot Acquisition Controller for the RADARSAT Spacecraft," Flight Mechanics Symposium, edited by Walls, D. M., NASA Goddard Space Flight Center, Greenbelt, MD, 1997, pp. 79-89.

[15] Chang, Y.-K., Lee, B.-H., and Kim, S.-J., "Momentum Wheel Start-Up Method for HAUSAT-2 Ultra-Small Satellite," Aerospace Science and Technology, Vol. 10, No. 2, March 2006, pp. 168-174. doi:10.1016/j.ast.2005.07.011

[16] Thyagarajan, K., Gupta, J. P., Goel, P. S., and Jayaraman, K., "University Small Satellite Program - ANUSAT," Acta Astronautica, Vol. 56, Nos. 1-2, Jan. 2005, pp. 89-97. doi:10.1016/j.actaastro.2004.09.038

[17] Santoni, F., and Zelli, M., "Passive Magnetic Attitude Stabilization of the UNISAT-4 Microsatellite," Acta Astronautica, Vol. 65, Nos. 5-6, Sept. 2009, pp. 792-803. doi:10.1016/j.actaastro.2009.03.012

[18] Avanzini, G., and Giulietti, F., "Magnetic Detumbling of a Rigid Spacecraft," Journal of Guidance, Control, and Dynamics, Vol. 35, No. 4, July 2012, pp. 1326-1334. doi: $10.2514 / 1.53074$

[19] Avanzini, G., De Angelis, E. L., and Giulietti, F., "Acquisition of a Desired Pure-Spin Condition for a Magnetically Actuated Spacecraft," Journal of Guidance, Control, and Dynamics, Vol. 36, No. 6, Nov. 2013, pp. $1816-1821$.

doi:10.2514/1.59364

[20] Cheon, Y.-J., Lee, S.-H., and Kin, J.-H., "Fully Magnetic Devices-Based Control for Gyroless Target Pointing of a Spinning Spacecraft," IEEE Aerospace And Electronic Systems Magazine, Vol. 46, No. 3, 2010, pp. 1484-1491. doi:10.1 109/TAES.2010.5545203

[21] Stickler, A. C., and Alfriend, K. T., "Elementary Magnetic Attitude Control System," Journal of Spacecraft and Rockets, Vol. 13, No. 5, 1976, pp. 282-287. doi:10.2514/3.57089

[22] Ravanbakhsh, A., and Franchini, S., "Multiobjective Optimization Applied to Structural Sizing of Low Cost University-Class Microsatellite Projects," Acta Astronautica, Vol. 79, Oct. 2012, pp. 212-220. doi:10.1016/j.actaastro.2012.04.011

[23] Sanz-Andrés, A., Meseguer, J., Perales, J. M., and Santiago-Prowald, J., "A Small Platform for Astrophysical Research Based on the UPM-SAT 1 Satellite oh the Universidad Politécnica de Madrid," Advances in Space Research, Vol. 31, No. 2, 2003, pp. 375-380. doi:10.1016/S0273-1177(02)00629-4

[24] De Manuel, C., Cubas, J., and Pindado, S., "On the Simulation of the UPMSat-2 Microsatellite Power," Proceedings of 10th European Space Power Conference, ESA Publications Division ESTEC, Noordwijk, The Netherlands, 2014.

[25] Wertz, J. R., Spacecraft Attitude Determination and Control, Kluwer, Dordrecht, The Netherlands, 1978, Chap. 15.

[26] Maus, S., Nga, C. R., Macmillan, S., and Thomson, A., "The US/UK World Magnetic Model for 2010-2015," National Oceanic and Atmospheric Administration, Technical Rept., National Environmental Satellite Data and Information Service/National Geophysical Data Center, 2010.

[27] Jensen, K. F., and Vinther, K., Attitude Determination and Control System for AAUSAT3, Aalborg Univ., Aalborg, Denmark, 2010.

[28] Khalil, H. K., Nonlinear Systellts, 3rd ed., Prentice-Hall, Upper Saddle River, NJ, 2002, Chap. 4.

[29] Kaplan, M., Modern Spacecraft Dynamics and Control, Wiley, New York, 1976, Chap. 2.

[30] Beer, P., and Johnson, E. R., Vector Mechanics for Engineers (Dynamics), McGraw-Hill, New York, 1977, Sec. 18.10. 